\title{
Neutrino Mass and Neutrinoless double beta decay in SO(10) GUT with Pati-Salam symmetry
}

\author{
M. Sruthilaya ${ }^{a}$, Rukmani Mohanta $^{a}$, Sudhanwa Patra $^{b}$ \\ ${ }^{a}$ School of Physics, University of Hyderabad, Hyderabad - 500046, India \\ ${ }^{b}$ Center of Excellence in Theoretical and Mathematical Sciences, \\ Siksha 'O' Anusandhan University, Bhubaneswar-751030, India \\ E-mail: msruthi28@gmail.com, rmsp@uohyd.ernet.in, sudha.astro@gmail.com
}

ABstract: We demonstrate how a class of non-supersymmetric $S O(10)$ GUT with asymmetric left-right theory $S U(2)_{L} \times U(1)_{R} \times U(1)_{B-L} \times S U(3)_{C}$ and Pati-Salam theory $S U(2)_{L} \times S U(2)_{R} \times S U(4)_{C}$ as intermediate symmetry breaking steps leads to successful gauge coupling unification satisfying proton decay constraints. The motivation behind this work is two fold: firstly to study the renormalization group evolution equations for gauge couplings by keeping right-handed neutral gauge boson $Z_{R}$ around LHC energy range leading interesting dilepton searches at collider while fixing charge partner of the gauge boson $W_{R}$ at very high scale; secondly to explain neutrino masses and associated lepton number violating process like neutrinoless double beta decay in three possible cases depending on how $S U(2)_{L} \times U(1)_{R} \times U(1)_{B-L} \times S U(3)_{C}$ breaks down to SM. The presence of Pati-Salam symmetry and Pati-Salam symmetry with D-parity (discrete left-right symmetry leading to $\left.g_{L}=g_{R}\right)$ at highest scale is to allow two gauge couplings and thereby ensuring precision unification for gauge couplings. We focus on neutrino mass and neutrinoless double beta decay for one particular case where $\mathrm{TeV}$ scale asymmetric left-right theory is spontaneously broken down to SM with non-zero VEV of both Higgs doublets with $B-L=-1$ and Higgs triplets with $B-L=2$. We include one extra fermion singlet per generation in order to implement gauged extended seesaw where light neutrino mass is governed by natural type-II seesaw mechanism whereas type-I seesaw contribution is exactly canceled out. Since light neutrino mass formula is independent of Dirac neutrino mass matrix, the value of Dirac neutrino mass is taken to be up-type quark mass matrix which is a characteristics of PatiSalam symmetry relating quarks with leptons. This large value of Dirac neutrino mass can contribute to neutrinoless double beta decay, non-unitarity effects in leptonic sector and lepton flavor violation. We present analytic relation for effective Majorana mass parameter and corresponding half-life arising from new physics contributions due to purely left-handed currents through exchange of heavy right-handed neutrinos and sterile neutrinos. We numerically estimate effective Majorana mass parameter and half-life vs. lightest neutrino mass and derive lower bound on lightest neutrino mass by saturating with experimental bounds like GERDA Phase-II, KamLANDZen and EXO.

Keywords: Neutrino Mass, Neutrinoless Double Beta Decay, Grand Unification 


\section{Contents}

1 Introduction 2

2 SO(10) GUT with Pati-Salam symmetry 4

2.1 RG evolution for the gauge couplings 5

2.2 Matching conditions for inverse fine structure constants: 6

2.3 Solutions for $M_{D_{P}}$ and $M_{U}$ by fixing $M_{Z_{R}}$ around $\mathrm{TeV}$ scale $\quad 7$

3 Grand Unification with intermediate $U(1)_{R} \times U(1)_{B-L} \rightarrow U(1)_{Y}$ breaking via Higgs doublets $H_{L, R} \quad \mathbf{8}$

3.1 Gauge coupling unification and different mass scales 8

$\begin{array}{llr}3.2 & \text { Fermion mass fitting } & 9\end{array}$

3.3 Comment on Neutrino Mass and Neutrinoless double beta decay 11

4 Grand Unification with intermediate $U(1)_{R} \times U(1)_{B-L} \rightarrow U(1)_{Y}$ breaking via Higgs triplets $\Delta_{L, R} \quad 12$

$\begin{array}{lll}4.1 & \text { Gauge coupling unification } & 12\end{array}$

$\begin{array}{lll}4.2 & \text { Fermion mass fitting } & 12\end{array}$

$\begin{array}{lll}4.3 & \text { Comment on Neutrino Mass } & 13\end{array}$

$\begin{array}{lll}4.4 \text { Comment on Neutrinoless double beta decay } & 14\end{array}$

5 Grand Unification with intermediate $U(1)_{R} \times U(1)_{B-L} \rightarrow U(1)_{Y}$ breaking $\begin{array}{ll}\text { via Higgs doublets and triplets } & \mathbf{1 5}\end{array}$

$\begin{array}{lll}5.1 & \text { Spontaneous symmetry breaking } & 15\end{array}$

$\begin{array}{ll}5.2 \text { Gauge coupling unification } & 15\end{array}$

$\begin{array}{lll}5.3 & \text { Fermion mass fitting } & 16\end{array}$

$\begin{array}{lll}5.4 & \text { Discussion on Neutrino Mass } & 18\end{array}$

5.5 Neutrinoless double beta decay from large light-heavy neutrino mixing 20

$\begin{array}{ll}5.6 & \text { Numerical result for } 0 \nu \beta \beta\end{array}$

$\begin{array}{llr}6 & \text { Conclusion } & 28\end{array}$

$\begin{array}{llr}7 & \text { Acknowledgement } & 29\end{array}$ 


\section{Introduction}

The Standard Model (SM) of particle physics, a well developed theory whose predictions are verified with high precision, seems to be the low energy regime of a more fundamental theory. There are some observations like tiny neutrino mass, existence of dark matter, baryon asymmetry etc., urge for physics beyond the standard model. In the SM all particles get their mass through Higgs mechanism and neutrinos are strictly massless due to the absence of right handed partner, a must entity to get mass through Higgs mechanism. Dark matter is a matter whose existence is known only through gravitational effect and none of the standard model particles can accommodate the observed dark matter density. Also the $\mathrm{CP}$ violation in the SM is insufficient to explain the matter-antimatter asymmetry of the Universe. All these indicate that particle content of the standard model has to be extended.

The neutrino mass can be explained if right-handed neutrinos are added to the SM. Since right handed neutrinos are singlets under SM symmetry group, they can have Majorana mass which violate $B-L$, an accidental symmetry of the SM by two units. A very high Majorana mass of right-handed neutrinos give rise to tiny mass of light neutrinos through Type-I seesaw mechanism [1-5] with not so small Yukawa coupling between Higgs and neutrinos. But in this case Majorana mass is an arbitrary parameter unlike Dirac mass which is originated by spontaneous breaking of electroweak symmetry. Majorana mass of right handed neutrinos can be generated exactly in the same way as mass of other particles if the SM gauge symmetry is extended by an $S U(2)_{R}$ symmetry and SM particle content by a Higgs which is a triplet under the new symmetry. Other than Type-I seesaw, there are many seesaw mechanisms to attain tiny active neutrino mass and all demand additional new particles to the SM particle content. Type-II seesaw [5-11] is one among them, which requires existence of three Higgs fields belonging to the triplet representation of $S U(2)_{L}$.

Left-right symmetric [12-15] models are examples of popular models where SM symmetry is extended by $S U(2)_{R}$ symmetry and right-handed neutrinos get Majorana mass when neutral component of right-handed Higgs triplets get vacuum expectation value. Both these models reveal parity is conserved at very high energies and is spontaneously broken at somewhere above the electroweak scale $[14,16]$, hence give the origin of parity violation in the standard model. The left-right symmetric model-when spontaneous symmetry breaking occurs at few $\mathrm{TeV}$-offers numerous weak interaction phenomenology with $\mathrm{TeV}$ scale spectrum of extra gauge bosons $W_{R}^{ \pm}, Z_{R}$, right-handed neutrinos and associated scalars. Keeping charged gauge boson $W_{R}$ and neutral gauge boson $Z_{R}$ within LHC limit, TeV scale left-right symmetry provides testable consequences for collider signals in the gauge sector [17-47] as well as Higgs sector [15, 48-64]. Many attempts have been made in the context of left-right symmetry with and without spontaneous D-parity breaking to give new physics contribtions to neutrinoless double beta decay $(0 \nu \beta \beta)$ [9, 65-93], low-energy charged lepton flavor violation (cLFV) [23, 69, 74, 80, 82, 84, 94-104] and electric dipole moment (EDM) [71, 77, 105-108]. A few attempts have also made in left-right symmetric model to accommodate stable component of dark matter [109-112, 112-114, 114-118].

Models based on Left-Right symmetry are also successful in explaining the existence 
of dark matter and baryon asymmetry of the Universe. The recent model discussed in Ref. [119] explains both baryon asymmetry accounting for matter-antimatter asymmetry of the universe and existence of dark matter based on Pati-Salam symmetry with four generations of fermions. In this model, fourth generation neutrino forms dark matter and its number density is shown to account for the major part of dark matter in the Universe. In [120] baryon asymmetry and existence of dark matter is explained by a model based on left-right symmetry and [109] shows $\mathrm{TeV}$ scale dark matter can be accommodated in the left-right symmetric model. Even though these models solve many problems of the standard model they do not explain why there are interactions with different strength and particles with a wide range of mass, a grand unified theory (GUT) is an answer to these questions. Grand Unified Theory (GUT) tells at very high energy all the three interactions of SM have same strength and because of different renormalization group evolution with energy due to the particle content of the model, end up with different strengths at present.

Standard model is a very successful theory at low energy and hence, any GUT candidate should have SM gauge group as its sub group so that SM will be retained at electroweak scale. Since the rank of SM gauge group is four, the rank of gauge group of GUT should be greater than or equal to four. Hence the smallest group which can be the gauge group of GUT is $S U(5)$ [121]. $S O(10), E_{6}$ etc. are other candidates for GUT gauge group. Along with explaining the experimental observations there are some predictions of GUT such as proton decay. Non super-symmetric $S U(5)$ GUT is ruled out as it predicts proton lifetime much below the experimental value whereas both $\mathrm{SO}(10)$ and $E_{6}$ predict correct proton life time in their non-supersymmetric version. Among many models, $S O(10)$ GUT is a promising theory as it unifies all the fermion content of SM along with right-handed neutrino in a 16 dimensional spinorial representation, thereby giving a common origin for their mass. There are a number of ways in which $\mathrm{SO}(10)$ can be broken to SM, which justify experimental observations and are unique by their predictions.

A GUT with Pati-Salam or Left-Right symmetries as intermediate symmetry will be of great interest. In models based on left-right symmetry or Pati-Salam symmetry, discrete parity symmetry breaks along with $S U(2)_{R}$ symmetry and embedding of these models in $\mathrm{SO}(10)$ GUT requires parity breaking scale to be very high to get the observed value of $\sin ^{2} \theta_{W}$ [122], hence any effect due to the right handed current will be highly suppressed which makes the theory un-testable. Introduction of D-parity, symmetry between left and right chiral fields in $S O(10)$ makes parity braking scale to decouple from that of $S U(2)_{R}$ symmetry [123-126]. In such models D-parity breaks at very high energy and $S U(2)_{R}$ at much lower energy, giving rise to a testable effect of right handed currents at energies accessible to current experiments.

In the present work, we consider a non-supersymmetric $S O(10)$ GUT with intermediate symmetry groups such as Pati-Salam symmetry $S U(2)_{L} \times S U(2)_{R} \times S U(4)_{C}$ and $\mathrm{TeV}$ scale asymmetric left-right theory $S U(2)_{L} \times U(1)_{R} \times U(1)_{B-L} \times S U(3)_{C}$ manifesting in an extra right-handed neutral gauge boson $Z_{R}$ which might be detected by ongoing search experiments at the Large Hadron Collider. Depending on spontaneous symmetry breaking of $S U(2)_{L} \times U(1)_{R} \times U(1)_{B-L} \times S U(3)_{C}$ down to SM, we study three different scenarios with particular choice of Higgs and comment on neutrino mass and lepton number violating 
processes like neutrinoless double beta decay accordingly. We focus on gauged extended seesaw mechanism for the case where asymmetric left-right symmetry breaks down to SM via both Higgs doublet plus triplet and the fermion sector comprises of usual quarks and leptons plus one sterile fermions per generation. The mass formula for light neutrinos is governed by type-II seesaw dominance while type-I seesaw is exactly canceled out in the diagonalization method. We plan to discuss neutrino mass and neutrinoless double beta decay within type-II seesaw dominance relating light and heavy mass eigenvalues assuming Dirac neutrino mass matrix equals to up-type quark mass matrix. We wish to derive bound on lightest neutrino mass from new physics contributions to neutrinoless double beta decay arising from exchange of right-handed neutrinos and sterile neutrinos.

The plan of the paper is organized as following way. In Sec-II we briefly discuss the symmetry breaking chain of $S O(10)$ with asymmetric left-right model and Pati-Salam symmetry as intermediate steps of breaking and present the one-loop RGEs for gauge couplings along with their matching conditions at different symmetry breaking scales and important mass relations. We discuss possible ways to implement the symmetry breaking of $U(1)_{R} \times U(1)_{B-L} \rightarrow U(1)_{Y}$, gauge coupling unification, neutrino mass and other low energy phenomenon in Sec-III, Sec-IV and Sec-V respectively. In Sec-III and Sec-IV, we examine gauge coupling unification and commented on neutrino mass and neutrinoless double beta decay. In Sec-V, we study successful gauge coupling unification with intermediate mass scales such that they generate neutrino mass via type-II seesaw mechanism. We emphasize this case with discussion on neutrinoless double decay and derive lower bounds on lightest neutrino mass by saturating experimental bounds. Towards end, we summarize our results and conclude in Sec-VI.

\section{$2 \mathrm{SO}(10)$ GUT with Pati-Salam symmetry}

The idea here is to discuss neutrino mass and associated lepton number violating process like neutrinoless double beta decay $(0 \nu \beta \beta)$ in a non-supersymmetric $S O(10)$ GUT. The popular symmetry breaking is $S O(10) \rightarrow \mathcal{G}_{S M}$ without having any intermediate symmetry breaking steps. There are also other symmetry breaking chain $S O(10) \rightarrow \mathcal{G}_{I} \rightarrow \mathcal{G}_{S M}$ where the intermediate symmetry breaking could be a three gauge groups theory like PatiSalam theory $S U(2)_{L} \times S U(2)_{R} \times S U(4)_{C}$ or four gauge groups theory like left-right theory $S U(2)_{L} \times S U(2)_{R} \times U(1)_{B-L} \times S U(3)_{C}$. Instead of manifest left-right symmetry group $S U(2)_{L} \times S U(2)_{R} \times U(1)_{B-L} \times S U(3)_{C}$, one may consider asymmetric left-right theory with gauge group $S U(2)_{L} \times U(1)_{R} \times U(1)_{B-L} \times S U(3)_{C}$ as possible intermediate symmetry breaking step where $S U(2)_{R}$ has broken down to $U(1)_{R}$ without breaking rank of the gauge group. One such symmetry breaking chain for $S O(10)$ GUT having asymmetric left-right theory as well as Pati-Salam symmetry as possible subgroups is given by

$$
S O(10) \stackrel{M_{U}}{\longrightarrow} \mathcal{G}_{2_{L} 2_{R} 4_{C} D} \stackrel{M_{D_{P}}}{\longrightarrow} \mathcal{G}_{2_{L} 2_{R} 4_{C}} \stackrel{M_{W_{R}}}{\longrightarrow} \mathcal{G}_{2_{L} 1_{R} 1_{B-L} 3_{C}} \stackrel{M_{Z_{R}}}{\longrightarrow} \mathcal{G}_{2_{L} 1_{Y} 3_{C}}(\mathrm{SM}) \stackrel{M_{W}}{\longrightarrow} \mathcal{G}_{1_{Q} 3_{C}},
$$


where we have defined

$$
\begin{aligned}
& \mathcal{G}_{2_{L} 2_{R} 4_{C} D} \equiv S U(2)_{L} \times S U(2)_{R} \times S U(4)_{C} \times D \\
& \mathcal{G}_{2_{L} 2_{R} 4_{C}} \equiv S U(2)_{L} \times S U(2)_{R} \times S U(4)_{C} \\
& \mathcal{G}_{2_{L} 1_{R} 1_{B-L} 3_{C}} \equiv S U(2)_{L} \times U(1)_{R} \times U(1)_{B-L} \times S U(3)_{C}, \\
& \mathcal{G}_{2_{L} 1_{Y} 3_{C}} \equiv S U(2)_{L} \times U(1)_{Y} \times S U(3)_{C} .
\end{aligned}
$$

In this work, we consider how the left-right asymmetric gauge group $S U(2)_{L} \times U(1)_{R} \times$ $U(1)_{B-L} \times S U(3)_{C}$ breaks down to the Standard Model gauge group $S U(2)_{L} \times U(1)_{Y} \times$ $S U(3)_{C}$ with the choice of Higgs field. We found that there are three possible ways to implement the symmetry breaking i.e., $U(1)_{R} \times U(1)_{B-L} \rightarrow U(1)_{Y}$ via

- Higgs doublet $H_{R}^{0}(1,1 / 2,-1,1)$ with $B-L$ charge -1

- Higgs triplet $\Delta_{R}^{0}(1,-1,2,1)$ with $B-L$ charge 2

- combination of Higgs doublet $H_{R}$ as well as Higgs triplet $\Delta_{R}$.

\subsection{RG evolution for the gauge couplings}

We study the RGEs for the gauge couplings in a non-supersymmetric SO(10) GUT with $\mathcal{G}_{2_{L} 1_{Y} 3_{C}}, \mathcal{G}_{2_{L} 1_{R} 1_{B-L} 3_{C}}, \mathcal{G}_{2_{L} 2_{R} 4_{C}}$ and $\mathcal{G}_{2_{L} 2_{R} 4_{C} D}$ as intermediate symmetry breaking steps where the evolution equations for running coupling constants at one-loop level is given by

$$
\mu \frac{\partial g_{i}}{\partial \mu}=\frac{a_{i}}{16 \pi^{2}} g_{i}^{3}
$$

which can be written in the form

$$
\frac{1}{\alpha_{i}\left(\mu_{2}\right)}=\frac{1}{\alpha_{i}\left(\mu_{1}\right)}-\frac{a_{i}}{2 \pi} \ln \left(\frac{\mu_{2}}{\mu_{1}}\right),
$$

where we denote $\alpha_{i}=g_{i}^{2} / 4 \pi$ as the fine structure constant for $i$-th gauge group and $\mu_{1}, \mu_{2}$ are two different energy scales with $\mu_{2}>\mu_{1}$. The master formula for one-loop betacoefficients $a_{i}$ determining the evolution of gauge couplings at one-loop order is given as

$$
a_{i}=-\frac{11}{3} \mathcal{C}_{2}(G)+\frac{2}{3} \sum_{R_{f}} T\left(R_{f}\right) \prod_{j \neq i} d_{j}\left(R_{f}\right)+\frac{1}{3} \sum_{R_{s}} T\left(R_{s}\right) \prod_{j \neq i} d_{j}\left(R_{s}\right) .
$$

In the above mentioned formula for $a_{i}, \mathcal{C}_{2}(G)$ represents the quadratic Casimir operator for the gauge bosons in their adjoint representation with values

$$
\mathcal{C}_{2}(G) \equiv\left\{\begin{array}{c}
N \text { if } S U(N) \\
0 \text { if } U(1)
\end{array}\right.
$$

Similarly, $T\left(R_{f}\right)$ and $T\left(R_{s}\right)$ are defined as the Dynkin indices of the irreducible representation for $R_{f, s}$ for a given fermion and scalar, respectively,

$$
T\left(R_{f, s}\right) \equiv\left\{\begin{array}{cl}
1 / 2 & \text { if } R_{f, s} \text { is fundamental, } \\
N & \text { if } R_{f, s} \text { is adjoint, } \\
0 & \text { if } R_{f, s} \text { is singlet }
\end{array}\right.
$$

Here $d\left(R_{f, s}\right)$ stands for the dimension of a given representation $R_{f, s}$ under all other $S U(N)$ gauge groups except the $i$-th gauge group. 


\subsection{Matching conditions for inverse fine structure constants:}

The inverse fine structure constants satisfy the following matching conditions at different scales. At the scale $\mu=M_{Z_{R}}, U(1)_{R} \times U(1)_{B-L}$ broken down to $U(1)_{Y}$ while $S U(3)_{C}$ and $S U(2)_{L}$ remain the same. Hence the matching conditions are given as

$$
\begin{aligned}
{\left[\alpha_{3 C}^{-1}\left(M_{Z_{R}}\right)\right]_{\mathcal{G}_{S M}} } & =\left[\alpha_{3 C}^{-1}\left(M_{Z_{R}}\right)\right]_{\mathcal{G}_{2113}}, \quad\left[\alpha_{2 L}^{-1}\left(M_{Z_{R}}\right)\right]_{\mathcal{G}_{S M}}=\left[\alpha_{2 L}^{-1}\left(M_{Z_{R}}\right)\right]_{\mathcal{G}_{2113}} \\
{\left[\alpha_{Y}^{-1}\left(M_{Z_{R}}\right)\right]_{\mathcal{G}_{S M}} } & =\left[\frac{3}{5} \alpha_{1 R}^{-1}\left(M_{Z_{R}}\right)+\frac{2}{5} \alpha_{B-L}^{-1}\left(M_{Z_{R}}\right)\right]_{\mathcal{G}_{2113}}
\end{aligned}
$$

Similarly at $\mu=M_{W_{R}}, S U(4)_{C}$ breaks to $S U(3) \times U(1)_{B-L}$ and $S U(2)_{R}$ breaks to $U(1)_{R}$ keeping $S U(2)_{R}$ intact. Hence, at this energy structure constants of $S U(3)_{C}$ and $U(1)_{B-L}$ are same and equal to that of $S U(4)_{C}$ while structure constants of $U(1)_{R}$ and $S U(2)_{L}$ of $\mathcal{G}_{2113}$ shares the same value with that of $S U(2)_{R}$ and $S U(2)_{L}$ of $\mathcal{G}_{224}$ respectively, i.e.,

$$
\begin{array}{ll}
{\left[\alpha_{3 C}^{-1}\left(M_{W_{R}}\right)\right]_{\mathcal{G}_{2113}}=\left[\alpha_{4 C}^{-1}\left(M_{W_{R}}\right)\right]_{\mathcal{G}_{224}},} & {\left[\alpha_{2 L}^{-1}\left(M_{W_{R}}\right)\right]_{\mathcal{G}_{2113}}=\left[\alpha_{2 L}^{-1}\left(M_{W_{R}}\right)\right]_{\mathcal{G}_{224}},} \\
{\left[\alpha_{1 R}^{-1}\left(M_{W_{R}}\right)\right]_{\mathcal{G}_{2113}}=\left[\alpha_{2 R}^{-1}\left(M_{W_{R}}\right)\right]_{\mathcal{G}_{224}},} & {\left[\alpha_{B-L}^{-1}\left(M_{W_{R}}\right)\right]_{\mathcal{G}_{2113}}=\left[\alpha_{4 c}^{-1}\left(M_{W_{R}}\right)\right]_{\mathcal{G}_{224}} .}
\end{array}
$$

From $\mu=M_{D_{P}}$ onwards D-parity is respected hence, the structure constants of $S U(2)_{R}$ and $S U(2)_{L}$ share same value for all energies above D-parity breaking scale $M_{D_{P}}$. Hence the matching conditions of structure constants at $M_{D_{P}}$ are

$$
\begin{aligned}
{\left[\alpha_{4 C}^{-1}\left(M_{D_{P}}\right)\right]_{\mathcal{G}_{224}} } & =\left[\alpha_{4 C}^{-1}\left(M_{D_{P}}\right)\right]_{\mathcal{G}_{224 D}}, \quad\left[\alpha_{2 L}^{-1}\left(M_{D_{P}}\right)\right]_{\mathcal{G}_{224}}=\left[\alpha_{2 L}^{-1}\left(M_{D_{P}}\right)\right]_{\mathcal{G}_{224 D}} \\
{\left[\alpha_{2 R}^{-1}\left(M_{D_{P}}\right)\right]_{\mathcal{G}_{224}} } & =\left[\alpha_{2 L}^{-1}\left(M_{D_{P}}\right)\right]_{\mathcal{G}_{224 D}}=\left[\alpha_{2 R}^{-1}\left(M_{D_{P}}\right)\right]_{\mathcal{G}_{224 D}} .
\end{aligned}
$$

At $\mu=M_{U}$ all the structure constants converge to a single one, the structure constant of $\mathrm{SO}(10)$, hence the matching conditions satisfy

$$
\left[\alpha_{2 L}^{-1}\left(M_{U}\right)\right]_{\mathcal{G}_{224 D}}=\left[\alpha_{2 R}^{-1}\left(M_{U}\right)\right]_{\mathcal{G}_{24 D}}=\left[\alpha_{4 C}^{-1}\left(M_{U}\right)\right]_{\mathcal{G}_{224 D}}=\left[\alpha_{10}^{-1}\left(M_{U}\right)\right]_{S O(10)} .
$$

We denote here the one loop beta coefficients in the mass range $M_{W}-M_{Z_{R}}$ as $a_{i}=$ $\left\{a_{2 L}, a_{Y}, a_{3 C}\right\}$, from $M_{Z_{R}}-M_{W_{R}}$ as $a_{i}^{\prime}=\left\{a_{2 L}^{\prime}, a_{1 R}^{\prime}, a_{B L}^{\prime}, a_{3 C}^{\prime}\right\}$, from $M_{W_{R}}-M_{D_{P}}$ as $a_{i}^{\prime \prime}=$ $\left\{a_{2 L}^{\prime \prime}, a_{2 R}^{\prime \prime}, a_{4 C}^{\prime \prime}\right\}$ and from $M_{D_{P}}-M_{U}$ as $a_{i}^{\prime \prime \prime}=\left\{a_{2 L}^{\prime \prime \prime}, a_{2 L}^{\prime \prime \prime}, a_{4 C}^{\prime \prime \prime}\right\}$. Solving Eq. (2.2) along with the matching conditions (2.7), (2.8), (2.9) and (2.10) gives the standard model gauge coupling constants as

$$
\begin{gathered}
\alpha_{3 C}^{-1}\left(M_{W}\right)=\left[\alpha_{10}^{-1}\left(M_{U}\right)\right]_{S O(10)}+\frac{a_{3 C}}{2 \pi} \ln \left(\frac{M_{Z_{R}}}{M_{W}}\right)+\frac{a_{3 C}^{\prime}}{2 \pi} \ln \left(\frac{M_{W_{R}}}{M_{Z_{R}}}\right) \\
+\frac{a_{4 C}^{\prime \prime}}{2 \pi} \ln \left(\frac{M_{D_{P}}}{M_{W_{R}}}\right)+\frac{a_{4 C}^{\prime \prime \prime}}{2 \pi} \ln \left(\frac{M_{U}}{M_{D_{P}}}\right) \cdot \\
\alpha_{2 L}^{-1}\left(M_{W}\right)=\left[\alpha_{10}^{-1}\left(M_{U}\right)\right]_{S O(10)}+\frac{a_{2 L}}{2 \pi} \ln \left(\frac{M_{Z_{R}}}{M_{W}}\right)+\frac{a_{2 L}^{\prime}}{2 \pi} \ln \left(\frac{M_{W_{R}}}{M_{Z_{R}}}\right) \\
+\frac{a_{2 L}^{\prime \prime}}{2 \pi} \ln \left(\frac{M_{D_{P}}}{M_{W_{R}}}\right)+\frac{a_{2 L}^{\prime \prime \prime}}{2 \pi} \ln \left(\frac{M_{U}}{M_{D_{P}}}\right) . \\
\alpha_{Y}^{-1}\left(M_{W}\right)=\left[\alpha_{10}^{-1}\left(M_{U}\right)\right]_{S O(10)}+\frac{a_{Y}}{2 \pi} \ln \left(\frac{M_{Z_{R}}}{M_{W}}\right)+\frac{1}{2 \pi}\left(\frac{3}{5} a_{1 R}^{\prime}+\frac{2}{5} a_{B L}^{\prime}\right) \ln \left(\frac{M_{W_{R}}}{M_{Z_{R}}}\right) \\
+\frac{1}{2 \pi}\left(\frac{3}{5} a_{2 R}^{\prime \prime}+\frac{2}{5} a_{4 C}^{\prime \prime}\right) \ln \left(\frac{M_{D_{P}}}{M_{W_{R}}}\right)+\frac{1}{2 \pi}\left(\frac{3}{5} a_{2 L}^{\prime \prime \prime}+\frac{2}{5} a_{4 C}^{\prime \prime \prime}\right) \ln \left(\frac{M_{U}}{M_{D_{P}}}\right) .
\end{gathered}
$$




\subsection{Solutions for $M_{D_{P}}$ and $M_{U}$ by fixing $M_{Z_{R}}$ around TeV scale}

At the energy scale $M_{W}$, the gauge coupling constants satisfy the following relations

$$
\frac{1}{\alpha_{e m}}\left(\sin ^{2} \theta_{W}-\frac{3}{8}\right)=\frac{5}{8}\left(\frac{1}{\alpha_{2 L}\left(M_{W}\right)}-\frac{1}{\alpha_{Y}\left(M_{W}\right)}\right)
$$

and

$$
8\left(\alpha_{s}^{-1}-\frac{3}{8} \alpha_{e m}^{-1}\right)=8 \alpha_{3 C}^{-1}-3 \alpha_{2 L}^{-1}-5 \alpha_{Y}^{-1}
$$

With the above conditions along with Eqs. (2.11) to (2.13) we obtain

$$
\mathcal{A}_{D} \ln \left(\frac{M_{D_{P}}}{M_{W}}\right)+\mathcal{A}_{U} \ln \left(\frac{M_{U}}{M_{W}}\right)=D_{0}
$$

where the different parameters in Eq. (2.16) are given as

$$
D_{0}=\frac{16 \pi}{\alpha_{e m}}\left(\sin ^{2} \theta_{W}-\frac{3}{8}\right)-\mathcal{A}_{Z_{R}} \ln \left(\frac{M_{Z_{R}}}{M_{W}}\right)-\mathcal{A}_{W_{R}} \ln \left(\frac{M_{W_{R}}}{M_{W}}\right),
$$

and

$$
\begin{aligned}
\mathcal{A}_{Z_{R}} & =\left(5 a_{2 L}-5 a_{Y}\right)-\left(5 a_{2 L}^{\prime}-3 a_{1 R}^{\prime}-2 a_{B-L}^{\prime}\right) \\
\mathcal{A}_{W_{R}} & =\left(5 a_{2 L}^{\prime}-3 a_{1 R}^{\prime}-2 a_{B-L}^{\prime}\right)-\left(5 a_{2 L}^{\prime \prime}-3 a_{2 R}^{\prime \prime}-2 a_{4 C}^{\prime \prime}\right) \\
\mathcal{A}_{D} & =\left(5 a_{2 L}^{\prime \prime}-3 a_{2 R}^{\prime \prime}-2 a_{4 C}^{\prime \prime}\right)-\left(2 a_{2 L}^{\prime \prime \prime}-2 a_{4 C}^{\prime \prime \prime}\right) \\
\mathcal{A}_{U} & =\left(2 a_{2 L}^{\prime \prime \prime}-2 a_{4 C}^{\prime \prime \prime}\right)
\end{aligned}
$$

Analogously, one can also obtain the relation

$$
\mathcal{B}_{D} \ln \left(\frac{M_{D_{P}}}{M_{W}}\right)+\mathcal{B}_{U} \ln \left(\frac{M_{U}}{M_{W}}\right)=D_{1},
$$

where

$$
D_{1}=16 \pi\left(\alpha_{s}^{-1}-\frac{3}{8} \alpha_{e m}^{-1}\right)-\mathcal{B}_{Z_{R}} \ln \left(\frac{M_{Z_{R}}}{M_{W}}\right)-\mathcal{B}_{W_{R}} \ln \left(\frac{M_{W_{R}}}{M_{W}}\right)
$$

and

$$
\begin{aligned}
\mathcal{B}_{Z_{R}} & =\left(8 a_{3 C}-3 a_{2 L}-5 a_{Y}\right)-\left(8 a_{3 C}^{\prime}-3 a_{2 L}^{\prime}-3 a_{2 R}^{\prime}-2 a_{B-L}^{\prime}\right), \\
\mathcal{B}_{W_{R}} & =\left(8 a_{3 C}^{\prime}-3 a_{2 L}^{\prime}-3 a_{1 R}^{\prime}-2 a_{B-L}^{\prime}\right)-\left(6 a_{4 C}^{\prime \prime}-3 a_{2 L}^{\prime \prime}-3 a_{2 R}^{\prime \prime}\right), \\
\mathcal{B}_{D} & =\left(6 a_{4 C}^{\prime \prime}-3 a_{2 L}^{\prime \prime}-3 a_{2 R}^{\prime \prime}\right)-\left(6 a_{4 C}^{\prime \prime \prime}-6 a_{2 L}^{\prime \prime \prime}\right), \\
\mathcal{B}_{U} & =\left(6 a_{4 C}^{\prime \prime \prime}-6 a_{2 L}^{\prime \prime \prime}\right) .
\end{aligned}
$$

Thus with Eqs. (2.16) and (2.19) we get the relations

$$
\begin{aligned}
& \ln \left(\frac{M_{U}}{M_{W}}\right)=\frac{D_{1} \mathcal{A}_{D}-D_{0} \mathcal{B}_{D}}{\mathcal{B}_{U} \mathcal{A}_{D}-\mathcal{A}_{U} \mathcal{B}_{D}} \\
& \ln \left(\frac{M_{D}}{M_{W}}\right)=\frac{D_{0} \mathcal{B}_{U}-D_{1} \mathcal{A}_{U}}{\mathcal{B}_{U} \mathcal{A}_{D}-\mathcal{A}_{U} \mathcal{B}_{D}} .
\end{aligned}
$$




\section{Grand Unification with intermediate $U(1)_{R} \times U(1)_{B-L} \rightarrow U(1)_{Y}$ breaking via Higgs doublets $H_{L, R}$}

The important stage of intermediate symmetry breaking $U(1)_{R} \times U(1)_{B-L} \rightarrow U(1)_{Y}$ is done via Higgs doublets $H_{L, R}$ with $B-L$ charge -1 at a scale of right-handed neutral gauge boson mass $M_{Z_{R}}$. We embed this intermediate left-right symmetry group $S U(2)_{L} \times$ $U(1)_{R} \times U(1)_{B-L} \times S U(3)_{C}$ into a $S O(10)$-GUT with Pati-Salam intermediate symmetry which can be best understood via the following way,

$$
\begin{aligned}
& S O(10) \\
& \downarrow\langle\eta(1,1,1)\rangle \subset 54_{H} \\
& S U(2)_{L} \times S U(2)_{R} \times S U(4)_{C} \times D \quad\left(g_{L}=g_{R}\right) \\
& \downarrow\langle\sigma(1,1,1)\rangle \subset 210_{H} \\
& S U(2)_{L} \times S U(2)_{R} \times S U(4)_{C} \quad\left(g_{L} \neq g_{R}\right) \\
& \downarrow\langle\Sigma(1,3,15)\rangle \subset 210_{H} \\
& S U(2)_{L} \times U(1)_{R} \times U(1)_{B-L} \times S U(3)_{C} \quad\left(g_{L} \neq g_{R}\right) \\
& \downarrow\left\langle H_{R}^{0}(1,1 / 2,-1,1)\right\rangle \subset 16_{H} \\
& S U(2)_{L} \times U(1)_{Y} \times S U(3)_{C} \\
& \downarrow\left\langle\phi\left(2, \frac{1}{2}, 1\right)\right\rangle \subset 10_{H} \\
& U(1)_{Q} \times S U(3)_{C} .
\end{aligned}
$$

At first, the GUT scale symmetry breaking $S O(10) \rightarrow \mathcal{G}_{2_{L} 2_{R} 4_{C} D}$ is achieved by giving non-zero vacuum expectation value to $\mathrm{D}$-parity even singlet $\eta(1,1,1) \subset 54_{H}$. As a result, the Higgs sector is symmetric under left-right invariance from D-parity scale $\left(M_{D_{P}}\right)$ to unification scale $\left(M_{U}\right)$ leading to $g_{L}=g_{R}$. The second step of symmetry breaking $\mathcal{G}_{2_{L} 2_{R} 4_{C} D} \rightarrow \mathcal{G}_{2_{L} 2_{R} 4_{C}}$ is done by assigning a non-zero VEV to D-parity odd singlet $\sigma(1,1,1) \subset 210_{H}$ which is part of $S O(10)$ GUT $[124,125]$. The next stage of symmetry breaking $\mathcal{G}_{2_{L} 2_{R} 4_{C}} \rightarrow \mathcal{G}_{2_{L} 1_{R} 1_{B-L} 3_{C}}$ is occurred by the neutral component of right-handed Higgs $\Sigma_{R}(1,3,15) \subset 210_{H}$. At this stage of symmetry breaking, $S U(2)_{R}$ breaks down to $U(1)_{R}$ without breaking the rank of the gauge group and the $W_{R}$ gets it mass around PatiSalam symmetry breaking scale i.e, at $M_{W_{R}}$. The important stage of symmetry breaking is happened by giving non-zero VEV to neutral component of right-handed Higgs doublet $H_{R}(1,1 / 2,-1,1) \subset(1,2, \overline{4}) \subset 16_{H}$. At this stage, the extra right-handed neutral gauge boson $Z_{R}$ gets its mass around few $\mathrm{TeV}$. The last step of symmetry breaking is done with SM Higgs doublet $\phi(2,1 / 2,1) \subset 16_{H}$ leading to fermions masses and mixing.

\subsection{Gauge coupling unification and different mass scales}

We study RGEs for gauge couplings for non-supersymmetric $S O(10)$ GUT with asymmetric left-right theory, Pati-Salam symmetry and Pati-Salam symmetry with D-parity as intermediate subgroups and the $\mathrm{TeV}$ scale asymmetric left-right theory spontaneously broken down to SM via Higgs doublet with $B-L$ charge -1 . The Higgs spectrum and the derived one-loop beta coefficients for this framework are presented in Table. 1. The unification plot 
demonstrating successful gauge coupling unification is displayed in Fig. 1 with intermediate mass scales as follows,

$$
M_{Z_{R}} \approx 5 \mathrm{TeV}, \quad M_{W_{R}} \approx 10^{8.3} \mathrm{GeV}, \quad M_{D_{P}} \approx 10^{15.4} \mathrm{GeV}, \quad M_{U} \approx 10^{16.1} \mathrm{GeV} .
$$

\begin{tabular}{|c|c|c|c|}
\hline Group & Range of masses & Higgs Content & $a_{i}$ values \\
\hline \multirow{2}{*}{$\mathcal{G}_{213}$} & $M_{W}-M_{Z_{R}}$ & $\Phi(2,1 / 2,1)_{10}$ & $a_{i}=\left(\begin{array}{c}-19 / 6 \\
41 / 10 \\
-7\end{array}\right)$ \\
\hline \multirow{2}{*}{$\mathcal{G}_{2113}$} & $M_{Z_{R}}-M_{W_{R}}$ & $\Phi_{1}(2,1 / 2,0,1)_{10}, \Phi_{2}(2,-1 / 2,0,1)_{10^{\prime}}$ & $a_{i}^{\prime}=\left(\begin{array}{c}-3 \\
53 / 12 \\
33 / 8 \\
-7\end{array}\right)$ \\
\hline \multirow{2}{*}{$\mathcal{G}_{224}$} & $M_{W_{R}}-M_{D_{P}}$ & $H_{R}(1,1 / 2,-1,1)$ & \\
& & $\Phi_{1}(2,2,1)_{10}, \Phi_{2}(2,2,1)_{10^{\prime}}$ & $a_{i}^{\prime \prime}=\left(\begin{array}{c}-8 / 3 \\
8(3) \\
-19 / 3(-25 / 3)\end{array}\right)$ \\
\hline \multirow{G}{*}{$\mathcal{G}_{224 D}$} & $M_{D_{P}}-M_{U}$ & $\Phi_{1}(2,2,1,1)_{10}, \Phi_{2}(2,2,1)_{10^{\prime}}$ & \\
& & $H_{L}(2,1,4)_{16}, H_{R}(1,2, \overline{4})_{16}$ & $a_{i}^{\prime \prime \prime}=\left(\begin{array}{c}8(3) \\
8(3) \\
-2(-6)\end{array}\right)$ \\
\hline
\end{tabular}

Table 1. Details of the Gauge groups present in the symmetry breaking pattern with corresponding energy ranges, Higgs spectrum and one loop beta coefficients. For the last stage of symmetry breaking to Standard model symmetry is implemented by Higgs doublet $H_{R}$. The one loop beta coefficients are derived from Pati-Salam scale onwards by taking complex (real) Higgs representation $(1,3,15)$ where the numbers within parenthesis are for real representation.

\subsection{Fermion mass fitting}

Since $S O(10)$ grand unified theory unifies matter (15 components of fermions in one generation of SM plus additional right-handed neutrinos) in a 16-dimensional spinor and contains left-right symmetry and Pati-Salam symmetry as its subgroups, it provides fermion masses and mixing as well as relates quarks and leptons. Here, we intend to discuss the minimal realization of the theory by adding different combination of Higgs representation and comment whether or not they can be realistic and predictive. We restrict ourselves to Yukawa interactions of fermions at renormalizable level. It is already established that $S O(10) \mathrm{GUT}$ with only Higgs $10_{H}$ is inconsistent with fermion masses and mixing as SM doublet contained in $10_{H}$ is $S U(4)_{C}$ singlet and thus, results same structure for quarks and leptons.

With $16_{F} \otimes 16_{F}=10 \oplus 120 \oplus 126$, it is possible to write down three Yukawa interaction terms using $10_{H}, 120_{H}$ and $126_{H}$. As $10_{H}$ alone can not explain fermion masses and mixing and within the present scenario with no Higgs triplets contained in $126_{H}$, we are left with the choice $10_{H}+120_{H}$. With $10_{H}$ and $120_{H}$, the Yukawa interaction Lagrangian is given 


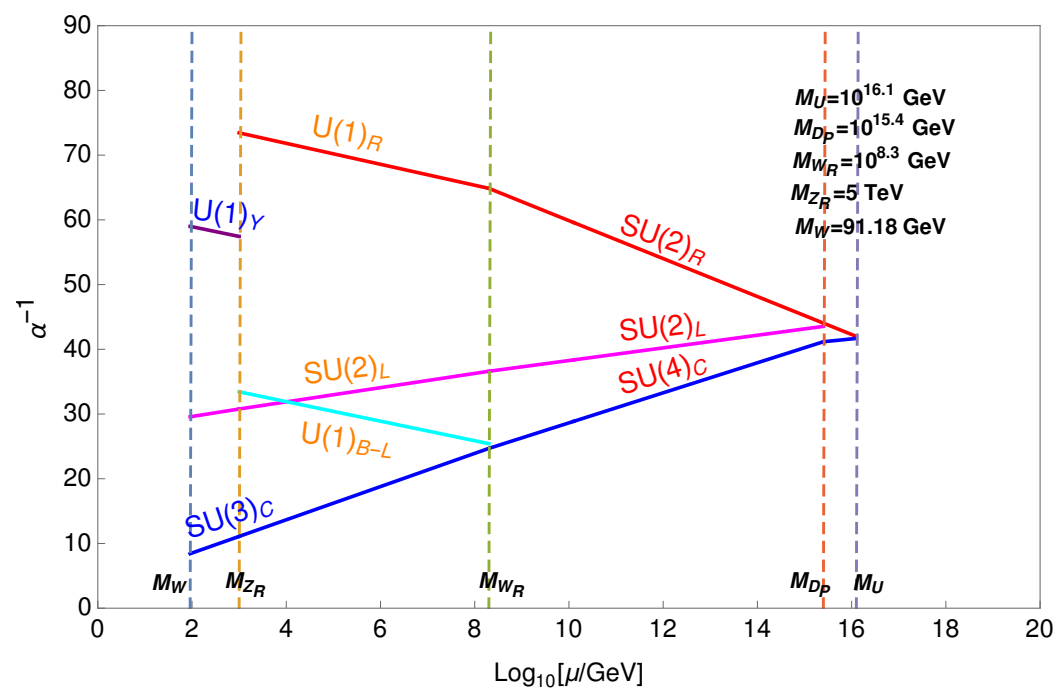

Figure 1. Evolution of inverse fine structure constants $\alpha_{i}^{-1}$ with variation of energy scale. Gauge coupling running demonstrating successful gauge unification within $\mathrm{SO}(10)$ GUT with intermediate asymmetric left-right gauge group and Pati-Salam symmetry breaking as intermediate symmetry breaking steps. We fix the right-handed neutral gauge boson scale around $5 \mathrm{TeV}$ and Pati-Salam breaking scale around $10^{8.3} \mathrm{GeV}$ while other mass scales like D-parity breaking scale and unification scale are predicted to be $M_{D_{P}} \approx 10^{15.4} \mathrm{GeV}$ and $M_{U} \approx 10^{16.1} \mathrm{GeV}$.

by

$$
\mathcal{L}_{Y}=16_{F}\left(Y_{10} 10_{H}+Y_{120} 120_{H}\right) 16_{F},
$$

with $Y_{10}=Y_{10}^{T}$ and $Y_{120}=-Y_{120}^{T}$. The Pati-Salam $S U(2)_{L} \times S U(2)_{R} \times S U(4)_{C}$ decomposition of $10_{H}$ and $120_{H}$ can be read as

$$
\begin{aligned}
& 10_{H}=(2,2,1) \oplus(1,1,6), \\
& 120_{H}=(2,2,1) \oplus(2,2,15) \oplus(1,1,10) \oplus(1,1, \overline{10}) \oplus(1,3,6) \oplus(3,1,6) .
\end{aligned}
$$

Using the non-zero VEVs of Higgs field contained in $10_{H}$ and $120_{H}$, the fermion masses read as [127],

$$
\begin{aligned}
& M_{d}=M_{0}+M_{2}, \quad M_{u}=c_{0} M_{0}+c_{2} M_{2}, \\
& M_{e}=M_{0}+c_{3} M_{2}, \quad M_{D}^{\nu}=c_{0} M_{0}+c_{4} M_{2},
\end{aligned}
$$


where

$$
\begin{aligned}
& M_{0}=Y_{10}\left\langle(2,2,1)_{10}^{d}\right\rangle, \\
& M_{2}=Y_{120}\left[\left\langle(2,2,1)_{120}^{d}\right\rangle+\left\langle(2,2,15)_{120}^{d}\right\rangle\right], \\
& c_{0} M_{0}=Y_{10}\left\langle(2,2,1)_{10}^{u}\right\rangle, \\
& c_{2} M_{2}=Y_{120}\left[\left\langle(2,2,1)_{120}^{u}\right\rangle+\left\langle(2,2,15)_{120}^{u}\right\rangle\right], \\
& c_{3}=\frac{\left\langle(2,2,1)_{120}^{d}\right\rangle-3\left\langle(2,2,15)_{120}^{d}\right\rangle}{\left\langle(2,2,1)_{120}^{d}\right\rangle+\left\langle(2,2,15)_{120}^{d}\right\rangle}, \\
& c_{4}=\frac{\left\langle(2,2,1)_{120}^{u}\right\rangle-3\left\langle(2,2,15)_{120}^{u}\right\rangle}{\left\langle(2,2,1)_{120}^{d}\right\rangle+\left\langle(2,2,15)_{120}^{d}\right\rangle} .
\end{aligned}
$$

In addition to this Dirac neutrino mass $M_{D}^{\nu}$, there could be two loop diagrams which can contribute to Majorana mass for right-handed neutrinos using $10_{H}, 16_{H}$ and $45_{V}$. The two loop contribution is proportional to $\propto(\alpha / \pi)^{2} Y_{10}\left(\left\langle 16_{H}\right\rangle^{2} / M_{G U T}\right)$ which is negligible in our present case with $\left\langle H_{R}\right\rangle \subset 16_{H}$ around few TeV. Thus, we have only dominant Dirac masses for light neutrinos. The sub-eV scale of Dirac neutrino mass can be achieved by suitable adjustment of different Yukawa couplings or fine tuning between $c_{0} M_{0}$ and $c_{4} M_{2}$. The details of the numerical fitting for fermion masses and mixing for three generation picture is rather involved and messy which is beyond the scope of this paper.

\subsection{Comment on Neutrino Mass and Neutrinoless double beta decay}

The fermion content of the asymmetric left-right model is given by

$$
\begin{aligned}
& \ell_{L}=\left(\begin{array}{c}
\nu_{L} \\
e_{L}
\end{array}\right) \sim(\mathbf{2}, \mathbf{0},-\mathbf{1}, \mathbf{1}), N_{R}(\mathbf{1}, \mathbf{1} / \mathbf{2},-\mathbf{1}, \mathbf{1}), \quad e_{R}(\mathbf{1},-\mathbf{1} / \mathbf{2},-\mathbf{1}, \mathbf{1}), \\
& q_{L}=\left(\begin{array}{l}
u_{L} \\
d_{L}
\end{array}\right) \sim\left(\mathbf{2}, \mathbf{0}, \frac{1}{3}, \mathbf{3}\right), u_{R}(\mathbf{1}, \mathbf{1} / \mathbf{2}, \mathbf{1} / \mathbf{3}, \mathbf{3}), \quad d_{R}(\mathbf{1},-\mathbf{1} / \mathbf{2}, \mathbf{1} / \mathbf{3}, \mathbf{3}) .
\end{aligned}
$$

The spontaneous symmetry breaking for asymmetric left-right model to SM is done via $\Phi_{1}(2,1 / 2,0,1), \Phi_{2}(2,-1 / 2,0,1)$ and $H_{R}(1,1 / 2,-1,1)$. No Majorana masses for left-handed as well as right-handed neutrinos are allowed due to absence of Higgs triplets. Thus, we have only Dirac masses for charged fermions,

$$
\begin{aligned}
& M_{u}=Y_{1} v_{1}+Y_{2} v_{2}^{*}, \quad M_{d}=Y_{1} v_{2}+Y_{2} v_{1}^{*}, \\
& M_{e}=Y_{3} v_{2}+Y_{4} v_{1}^{*},
\end{aligned}
$$

as well as for neutrinos

$$
M_{D}^{\nu} \equiv M_{D}=Y_{3} v_{1}+Y_{4} v_{2}^{*},
$$

where, $v_{1}\left(v_{2}\right)$ is the vacuum expectation value for $\Phi_{1}\left(\Phi_{2}\right)$ and $Y_{i}$ are the corresponding Yukawa couplings. The sub-eV scale of light active neutrinos is explained by this Dirac neutrino mass by adjusting these couplings and VEVs. Since there is no Majorana mass for neutrinos there is no lepton number violation present in the model where symmetry breaking of asymmetric left-right models is implemented with Higgs doublets. As a result of this, the lepton number violating process like neutrinoless double beta decay is absent. 


\section{Grand Unification with intermediate $U(1)_{R} \times U(1)_{B-L} \rightarrow U(1)_{Y}$ breaking via Higgs triplets $\Delta_{L, R}$}

In this section we discuss the case where breaking of $U(1)_{R} \times U(1)_{B-L}$ to $U(1)_{Y}$ is implemented by nonzero VEV of $\Delta_{R}(1,1,-2,1) \subset(1,3, \overline{10}) \subset 126_{H}$, the neutral component of right-handed Higgs triplet. It has a very different representation compared to $H_{R}(1,1 / 2,-1,1)$ under all symmetry groups present at different stages of symmetry breaking, hence affects RG evolution considerably. Moreover $\Delta_{R}$ belongs to higher dimensional representations of all symmetry groups other than $S U(2)_{L}$ which helps to lower the PatiSalam and $D$ parity breaking scale down to $10^{5} \mathrm{GeV}$ and $10^{12} \mathrm{GeV}$ respectively. A lower $D$ parity breaking scale ensures a sizable Type-II seesaw contribution to light neutrino mass. A sizable Type-II seesaw correction to neutrino mass makes it independent of quark mass whereas in $S O(10)$ grand unified theories Dirac neutrino mass and up-type quark mass have same origin. Breaking of $S U(2)_{R}$ symmetry at lower scale allows right handed currents to have detectable effects in low energy phenomenon such as neutrinoless double beta decay.

\subsection{Gauge coupling unification}

We study RGEs for gauge couplings for non-supersymmetric $S O(10)$ GUT with asymmetric left-right theory, Pati-Salam symmetry and Pati-Salam symmetry with D-parity as intermediate subgroups and the $\mathrm{TeV}$ scale asymmetric left-right theory spontaneously broken down to SM via Higgs triplet with $B-L$ charge 2 . The Higgs content and one-loop beta coefficients at various stages of symmetry breaking are given in Table. 2. The unification plot demonstrating successful gauge coupling unification is displayed in Fig. 2 with intermediate mass scales as follows,

$$
M_{Z_{R}} \approx 5 \mathrm{TeV}, \quad M_{W_{R}} \approx 10^{5} \mathrm{GeV}, \quad M_{D_{P}} \approx 10^{12.5} \mathrm{GeV}, \quad M_{U} \approx 10^{16.15} \mathrm{GeV} .
$$

\subsection{Fermion mass fitting}

We need $\Delta_{R} \subset 126_{H}, \Phi_{1} \subset 10_{H}$ and $\Phi_{1} \subset 10_{H}^{\prime}$ for spontaneous symmetry breaking of asymmetric left-right symmetry breaking. The relevant Yukawa Lagrangian at $\mathrm{SO}(10)$ level relevant for fermion masses and mixing is given by

$$
\mathcal{L}_{Y}=16_{F}\left(Y_{10} 10_{H}+Y_{10^{\prime}} 10_{H}^{\prime}+Y_{126} 126_{H}\right) 16_{F},
$$

with $Y_{10}=Y_{10}^{T}, Y_{10^{\prime}}=Y_{10^{\prime}}^{T}$ and $Y_{126}=Y_{126}^{T}$. The Pati-Salam $S U(2)_{L} \times S U(2)_{R} \times S U(4)_{C}$ decomposition of $10_{H}$ and $126_{H}$ is given by

$$
\begin{aligned}
& 10_{H}=(2,2,1) \oplus(1,1,6), \\
& 10_{H}^{\prime}=(2,2,1) \oplus(1,1,6), \\
& 126_{H}=(2,2,15) \oplus(3,1,10) \oplus(1,3, \overline{10}) \oplus(1,1,6) .
\end{aligned}
$$




\begin{tabular}{|c|c|c|c|}
\hline Group & Range of masses & Higgs Content & $a_{i}$ values \\
\hline $\mathcal{G}_{213}$ & $M_{W}-M_{2 R}$ & $\Phi(2,1 / 2,1)_{10}$ & $a_{i}=\left(\begin{array}{c}-19 / 6 \\
41 / 10 \\
-7\end{array}\right)$ \\
\hline $\mathcal{G}_{2113}$ & $M_{2 R}-M_{W_{R}}$ & $\begin{array}{c}\Delta_{R}(1,1,-2,1)_{126} \\
\oplus \Phi_{1}(2,1 / 2,0,1)_{10} \\
\oplus \Phi_{2}(2,-1 / 2,0,1)_{10^{\prime}}\end{array}$ & $a_{i}^{\prime}=\left(\begin{array}{c}-3 \\
14 / 3 \\
9 / 2 \\
-7\end{array}\right)$ \\
\hline $\mathcal{G}_{224}$ & $M_{W_{R}}-M_{D_{P}}$ & $\begin{array}{c}\Sigma_{R}(1,3,15)_{210} \\
\oplus \Delta_{R}(1,3, \overline{10})_{126} \\
\oplus \Phi_{1}(2,2,1)_{10} \\
\oplus \Phi_{2}(2,2,1)_{10^{\prime}}\end{array}$ & $a_{i}^{\prime \prime}=\left(\begin{array}{c}-8 / 3 \\
42 / 3(9) \\
-11 / 3(-17 / 3)\end{array}\right)$ \\
\hline $\mathcal{G}_{224 D}$ & $M_{D_{P}}-M_{U}$ & 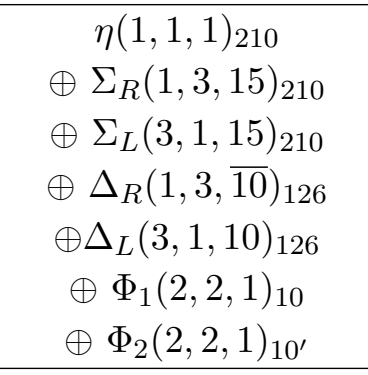 & $a_{i}^{\prime \prime \prime}=\left(\begin{array}{c}42 / 3(9) \\
42 / 3(9) \\
10 / 3(-2 / 3)\end{array}\right)$ \\
\hline
\end{tabular}

Table 2. Higgs spectrum with Higgs triplet to break $\mathcal{G}_{2113}$ to $\mathcal{G}_{S M}$ and corresponding one-loop beta coefficients at various energy ranges in the symmetry breaking chain. Here one loop beta coefficients are derived from Pati-Salam scale onwards by taking complex (real) Higgs representation $(1,3,15)$ where the numbers within parenthesis are for real representation.

With $10_{H}, 10_{H}^{\prime}$ and $126_{H}$, the fermion masses are given by

$$
\begin{aligned}
& M_{d}=v_{d}^{10} Y_{10}+v_{d}^{10^{\prime}} Y_{10^{\prime}}+v_{d}^{126} Y_{126}, \\
& M_{e}=v_{d}^{10} Y_{10}+v_{d}^{10^{\prime}} Y_{10^{\prime}}-3 v_{d}^{126} Y_{126}, \\
& M_{L}=v_{L} Y_{126}^{10} Y_{10}+v_{u}^{10^{\prime}} Y_{10^{\prime}}+v_{u}^{126} Y_{126}, \\
& M_{R}=v_{R} Y_{126} .
\end{aligned}
$$

We can ignore the type-II seesaw contribution $m_{\nu}^{I I}=f v_{L} \propto f \beta v^{2} v_{R} /\left(M^{\prime} M_{D_{P}}\right)$ (with large D-parity breaking scale $\left.M_{D_{P}}\right)$ as compared to type-I seesaw contribution $m_{\nu}^{I}=$ $-M_{D}^{\nu} M_{R}^{-1} M_{D}^{\nu^{T}}$. For $\mathrm{TeV}$ scale $M_{R}$, we need small value of $M_{D}$ which can be obtained with fine tuning the Yukawa couplings although the details is beyond the scope of this paper.

\subsection{Comment on Neutrino Mass}

A pleasant situation arises in which the spontaneous symmetry breaking is done by assigning a non-zero VEV to the neutral component of right-handed Higgs triplet $\Delta_{R}$ carrying $B-L$ charge 2. The left-right invariance demands the existence of left-handed Higgs triplet $\Delta_{L}$. The Majorana masses for left-handed as well as right-handed neutrinos are generated via 


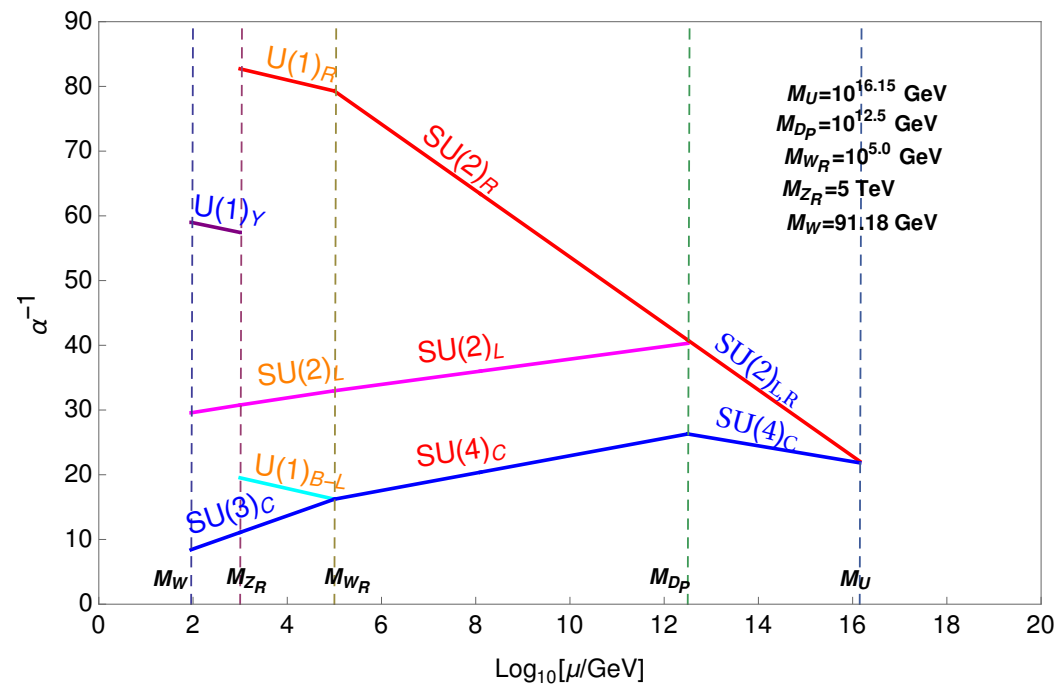

Figure 2. Evolution of inverse fine structure constants $\alpha_{i}^{-1}$ with variation of energy scale. We fix the right-handed neutral gauge boson scale around $5 \mathrm{TeV}$ and Pati-Salam breaking scale around $10^{5} \mathrm{GeV}$ while other mass scales like D-parity breaking scale and unification scale are predicted by RGEs. The D-parity breaking scale $M_{D_{P}}$ is found to be $10^{12.5} \mathrm{GeV}$ leading to induced $\mathrm{VEV}$ for scalar triplet and type-II seesaw contribution to light neutrino mass as $m_{\nu}^{I I}=f v_{L} \approx$ $\mathcal{O}(1) \cdot v^{2} \cdot v_{R} /\left(M^{\prime} \cdot M_{D_{P}}\right) \simeq 10^{-3} \mathrm{eV}$ while fixing $M^{\prime} \approx 10^{8} \mathrm{GeV}$. Thus, the type-I contributions to light neutrino mass is dominant than the type-II seesaw contribution having TeV scale of righthanded neutrinos.

non-zero VEV of $\Delta_{L}$ and $\Delta_{R}$ and thus violate lepton number by two units. We can write down the relevant interaction terms connecting scalar triplets and leptons as follows,

$$
-\mathcal{L}_{\text {Yuk }} \supset f_{i j}\left[\overline{\left(\ell_{L i}\right)^{c}} \ell_{L j} \Delta_{L}+\overline{\left(\ell_{R i}\right)^{c}} \ell_{R j} \Delta_{R}\right]+\text { h.c. . }
$$

The Higgs bidoublets will result Dirac neutrino mass matrix connecting both left- and righthanded neutrinos. The Majorana mass terms $M_{L}$ and $M_{R}$ arise due to presence of scalar triplets. Thus, the neutral lepton mass matrix in the basis $\left(\nu_{L}, N_{R}^{c}\right)$ reads as

$$
\mathcal{M}_{\nu}=\left(\begin{array}{ll}
M_{L} & M_{D} \\
M_{D}^{T} & M_{R}
\end{array}\right),
$$

The resulting light neutrino mass is governed by type-I and type-II seesaw formula as

$$
m_{\nu}=-M_{D} M_{R}^{-1} M_{D}^{T}+M_{L}=m_{\nu}^{I}+m_{\nu}^{I I},
$$

where, $m_{\nu}^{I}\left(m_{\nu}^{I I}\right)$ is for canonical type-I (type-II) seesaw contribution to light neutrino masses

$$
m_{\nu}^{I}=-M_{D} M_{R}^{-1} M_{D}^{T}, \quad m_{\nu}^{I I}=f v_{L}=f\left\langle\Delta_{L}^{0}\right\rangle .
$$

\subsection{Comment on Neutrinoless double beta decay}

Due to presence of Majorana nature of neutrinos in the present scenario, we have lepton number violating process like smoking-gun same-sign dilepton signatures at LHC as well 
as neutrinoless double beta decay in low energy experiments (for details refer to Refs [17, $20,23,28,35,37,67,69,70,72-75,78,100,101,128-133])$. In the context of neutrinoless double beta decay, many works consider either type-I seesaw dominance or type-II seesaw dominance within manifest left-right symmetric model, in left-right symmetric model with spontaneous D-parity breaking making unequal gauge couplings $g_{L} \neq g_{R}$. In most of these analysis, the left-right neutrino mixing (active-sterile neutrino mixing) is very much suppressed and as a result of this, the cross-section of heavy neutrino production and other lepton number violating processes at LHC are very much suppressed.

\section{Grand Unification with intermediate $U(1)_{R} \times U(1)_{B-L} \rightarrow U(1)_{Y}$ breaking via Higgs doublets and triplets}

\subsection{Spontaneous symmetry breaking}

Here the intermediate symmetry breaking $U(1)_{R} \times U(1)_{B-L} \rightarrow U(1)_{Y}$ is implemented with non-zero VEVs of both Higgs doublets $H_{R} \subset 16_{H}$ and triplets $\Delta_{R} \subset 126_{H}$ around few TeV scale. Then we embed this intermediate breaking step in a non-supersymmetric $S O(10)$ GUT with Pati-Salam symmetry and Pati-Salam symmetry with D-parity as two other intermediate symmetry breaking steps. The RG evolution equations for gauge coupling constants are almost similar to that in the previous section where $U(1)_{R} \times U(1)_{B-L}$ symmetry is broken by $\Delta_{R}$ alone. The only difference is the one-loop beta coefficients where both Higgs doublets and triplets contribute differently.

With the inclusion of extra sterile singlet neutrinos along with usual left- and righthanded neutrinos, the light neutrino mass formula is governed by extended type-II seesaw with the combination of $\Delta_{R}$ and $H_{R}$. In this case light neutrinos get mass only through Type-II seesaw even though right handed neutrinos get both Dirac and Majorana masses. The canonical type-I seesaw contribution to light neutrino mass is completely canceled out and thereby allow large value of Dirac neutrino mass which we will discuss in detail in the subsequent discussions. Within $S O(10)$ GUT with Pati-Salam intermediate symmetry it is not possible to have vanishing Dirac neutrino mass as Pati-Salam symmetry relates both up-type quark and Dirac neutrino mass matrices with each other. Since both Dirac and Majorana masses of right-handed neutrinos are independent of light neutrino mass, the structure of Dirac and Majorana masses may provide the scope of successful Leptogenesis to explain the Baryon Asymmetry of Universe.

\subsection{Gauge coupling unification}

We study RGEs for gauge couplings for non-supersymmetric $S O(10)$ GUT with asymmetric left-right theory, Pati-Salam symmetry and Pati-Salam symmetry with D-parity as intermediate subgroups and the $\mathrm{TeV}$ scale asymmetric left-right theory spontaneously broken down to SM via Higgs triplet with $B-L$ charge 2 . The Higgs content and one-loop beta coefficients at various stages of symmetry breaking are given in Table. 3. The unification plot demonstrating successful gauge coupling unification is displayed in Fig. 3 with intermediate 
mass scales as follows,

$$
M_{Z_{R}} \approx 5 \mathrm{TeV}, \quad M_{W_{R}} \approx 10^{5} \mathrm{GeV}, \quad M_{D_{P}} \approx 10^{12.3} \mathrm{GeV}, \quad M_{U} \approx 10^{16.1} \mathrm{GeV} .
$$

\begin{tabular}{|c|c|c|c|}
\hline Group & Range of masses & Higgs Content & $a_{i}$ values \\
\hline $\mathcal{G}_{213}$ & $M_{W}-M_{Z_{R}}$ & $\Phi(2,1 / 2,1)_{10}$ & $a_{i}=\left(\begin{array}{c}-19 / 6 \\
41 / 10 \\
-7\end{array}\right)$ \\
\hline $\mathcal{G}_{2113}$ & $M_{Z_{R}}-M_{W_{R}}$ & $\begin{array}{c}\Phi_{1}(2,1 / 2,0,1)_{10}, \Phi_{2}(2,-1 / 2,0,1)_{10^{\prime}} \\
\Delta_{R}(1,1,2,1)_{126}, H_{R}(1,1 / 2,-1,1)\end{array}$ & $a_{i}^{\prime}=\left(\begin{array}{c}-3 \\
57 / 12 \\
37 / 8 \\
-7\end{array}\right)$ \\
\hline $\mathcal{G}_{224}$ & $M_{W_{R}}-M_{D}$ & $\begin{array}{c}\Phi_{1}(2,2,1)_{10}, \Phi_{2}(2,2,1)_{10^{\prime}} \\
\Delta_{R}(1,3, \overline{10})_{126}, H_{R}(1,2, \overline{4})_{16} \\
\Sigma_{R}(1,3,15)_{210}\end{array}$ & $a_{i}^{\prime \prime}=\left(\begin{array}{c}-8 / 3 \\
44 / 3(29 / 3) \\
-10 / 3(-16 / 3)\end{array}\right)$ \\
\hline $\mathcal{G}_{224 D}$ & $M_{D}-M_{U}$ & $\begin{array}{c}\Phi_{1}(2,2,1)_{10}, \Phi_{2}(2,2,1)_{10^{\prime}} \\
\Delta_{R}(1,3, \overline{10})_{126}, \Delta_{L}(3,1,10)_{126} \\
H_{R}(1,2, \overline{4})_{16}, H_{L}(2,1,4)_{16} \\
\Sigma_{R}(1,3,15)_{210}, \Sigma_{L}(3,1,15)_{210} \\
\eta(1,1,1)_{210} \\
\end{array}$ & $a_{i}^{\prime \prime \prime}=\left(\begin{array}{c}44 / 3(29 / 3) \\
44 / 3(29 / 3) \\
4(0)\end{array}\right)$ \\
\hline
\end{tabular}

Table 3. Higgs spectrum with Higgs triplet and doublet to break $\mathcal{G}_{2113}$ to $\mathcal{G}_{S M}$ and corresponding one loop beta coefficients at various energy ranges in the symmetry breaking chain. The one loop beta coefficients are derived from Pati-Salam scale onwards by taking complex (real) Higgs representation $(1,3,15)$ where the numbers within parenthesis are for real representation.

\subsection{Fermion mass fitting}

We need both triplet $\Delta_{R} \subset 126_{H}$ as well as doublet $H_{R} \subset 16_{H}$ along with two bidoublets $\Phi_{1} \subset 10_{H}$ and $\Phi_{1} \subset 10_{H}^{\prime}$ for spontaneous symmetry breaking of asymmetric left-right symmetry breaking. Under Pati-Salam symmetry $S U(2)_{L} \times S U(2)_{R} \times S U(4)_{C}$, the quarks and leptons of $i$-th generation put in a single representation,

$$
F_{L(R)}=\left(\begin{array}{llll}
u_{r} & u_{g} & u_{b} & \nu_{e} \\
d_{r} & d_{g} & d_{b} & e
\end{array}\right)_{L(R)}
$$

Here $F_{L} \equiv(2,1,4), F_{R} \equiv(1,2, \overline{4})$ and $F_{L} \oplus F_{R}$ forms $16_{F}$ spinorial representation of $\mathrm{SO}(10)$. We know $(2,1,4) \otimes(1,2, \overline{4})=(2,2,1) \oplus(2,2,15)$, the Dirac masses for quarks and leptons of the form $Y \overline{F_{L}} \Phi F_{R}$ are generated through non-zero VEVs of $(2,2,1)$ of $10_{H}$ and $10_{H}^{\prime},(2,2,15)$ of $126_{H}$. Since $(2,2,1) \subset 126_{H}$ is very small, we need two bidoublets contained in $10_{H}$ and $10_{H}^{\prime}$ for correct charged fermion masses and mixing. On the other hand, the non-zero VEV of $(3,1,10)$ and $(1,3, \overline{10})$ give Majorana masses for left-handed and right-handed neutrinos. 


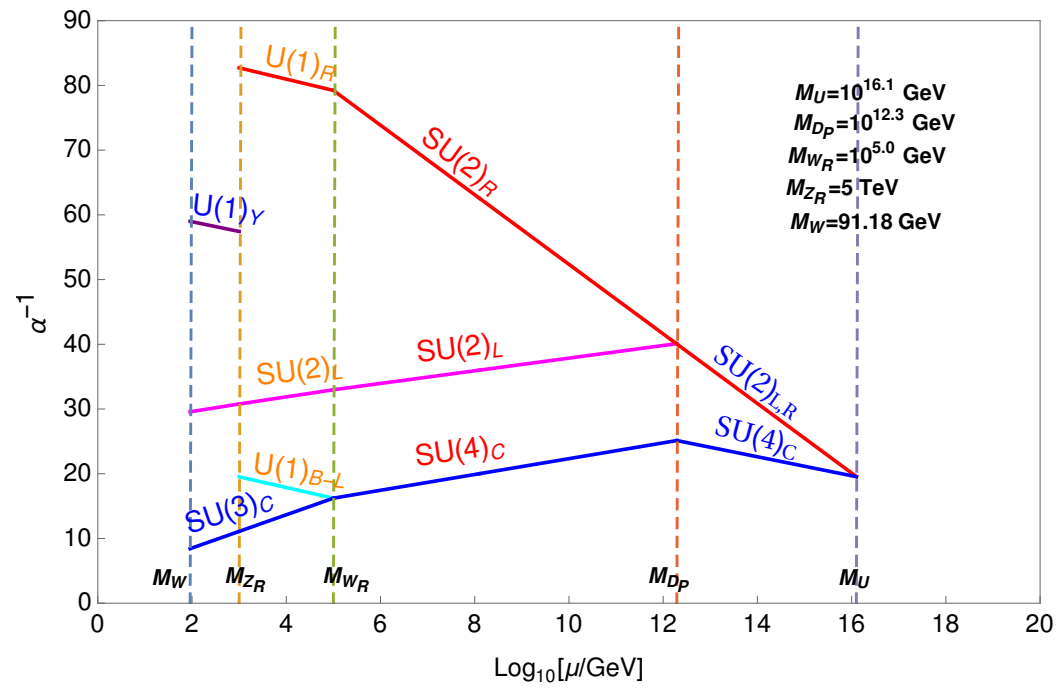

Figure 3. Evolution of inverse fine structure constants $\alpha_{i}^{-1}$ with variation of energy scale. We fix the right-handed neutral gauge boson scale around $5 \mathrm{TeV}$ and Pati-Salam breaking scale around $10^{5} \mathrm{GeV}$ while other mass scales like D-parity breaking scale and unification scale are predicted by RGEs. The D-parity breaking scale $M_{D_{P}}$ is found to be $10^{12.13} \mathrm{GeV}$ leading to induced VEV for scalar triplet and type-II seesaw contribution to light neutrino mass as $m_{\nu}^{I I}=$ $f v_{L} \approx \mathcal{O}(1) \cdot v^{2} \cdot v_{R} /\left(M^{\prime} \cdot M_{D_{P}}\right) \simeq 10^{-3}-10^{-2} \mathrm{eV}$ while fixing $M^{\prime} \approx 10^{8} \mathrm{GeV}$.

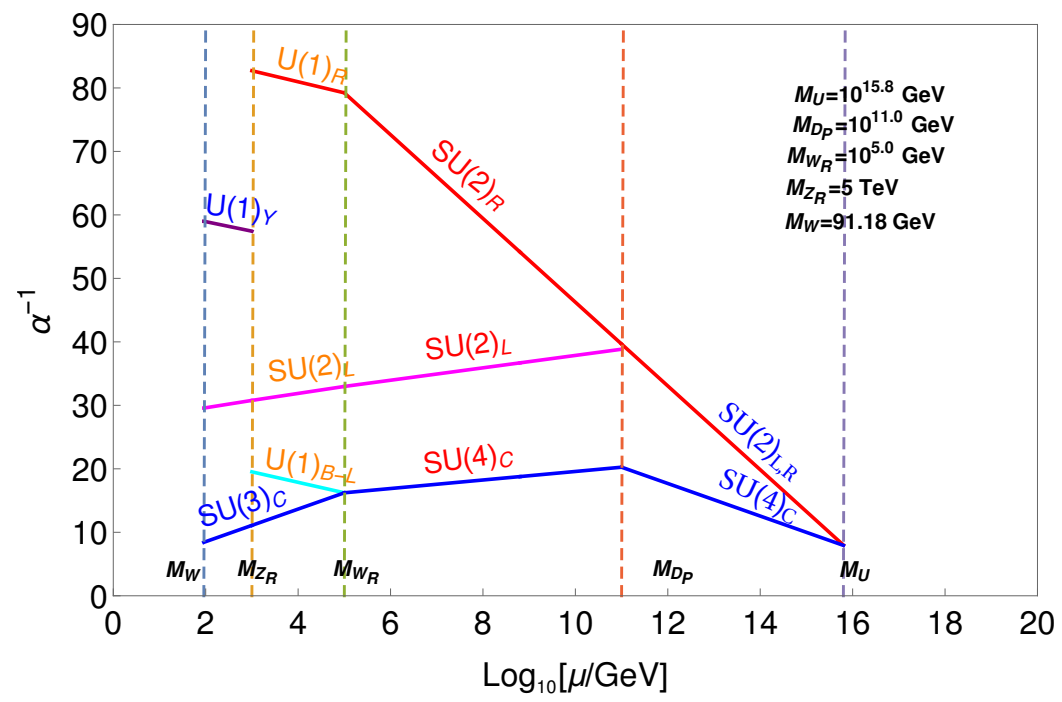

Figure 4. We demonstrate how one can lower the D-parity breaking scale by introducing another scalar triplet contained in $(1,3, \overline{10})$ from Pati-Salam symmetry breaking scale onwards which may enhance the scale of induced VEV of left-handed scalar triplet and the type-II seesaw contribution. In the presence if additional fermion singlet per generation, the type-I seesaw contribution is exactly canceled out leading to light neutrino masses via natural type-II seesaw dominance with large value of Dirac neutrino mass which gives observable effects. 
The $\mathrm{SO}(10)$ Yukawa Lagrangian for fermion masses is given by

$$
\mathcal{L}_{Y}=16_{F}\left(Y_{10} 10_{H}+Y_{10^{\prime}} 10_{H}^{\prime}+Y_{126} 126_{H}\right) 16_{F},
$$

with $Y_{10}=Y_{10}^{T}, Y_{10^{\prime}}=Y_{10^{\prime}}^{T}$ and $Y_{126}=Y_{126}^{T}$. The Pati-Salam $S U(2)_{L} \times S U(2)_{R} \times S U(4)_{C}$ decomposition of $10_{H}$ and $126_{H}$ is given by

$$
\begin{aligned}
& 10_{H}=(2,2,1) \oplus(1,1,6), \\
& 10_{H}^{\prime}=(2,2,1) \oplus(1,1,6), \\
& 16_{H}=(2,1,4) \oplus(1,2, \overline{4}), \\
& 126_{H}=(2,2,15) \oplus(3,1,10) \oplus(1,3, \overline{10}) \oplus(1,1,6) .
\end{aligned}
$$

The resulting fermion masses is given by

$$
\begin{aligned}
& M_{d}=v_{d}^{10} Y_{10^{\prime}}+v_{d}^{126} Y_{126}, \quad M_{u}=v_{u}^{10} Y_{10}+v_{u}^{126} Y_{126}, \\
& M_{e}=v_{d}^{10} Y_{10^{\prime}}-3 v_{d}^{126} Y_{126}, \\
& M_{L}=v_{L} Y_{126}, \\
& M_{R}=v_{R} Y_{126} .
\end{aligned}
$$

It is convenient to express $Y_{10}$ and $Y_{126}$ in terms of $M_{d}$ and $M_{e}[134,135]$ as follows,

$$
\begin{aligned}
& M_{u}=f_{u}\left[(3+r) M_{d}+(1-r) M_{e}\right], \\
& M_{D}^{\nu}=f_{u}\left[3(1-r) M_{d}+(1+3 r) M_{e}\right],
\end{aligned}
$$

where

$$
f_{u}=\frac{1}{4} \frac{v_{u}^{10}}{v_{d}^{10}}, \quad r=\frac{v_{d}^{10}}{v_{u}^{10}} \frac{v_{u}^{126}}{v_{d}^{126}} .
$$

The light neutrino mass formula is governed by type-II seesaw mechanism, $m_{\nu}^{I I}=M_{L}$ while type-I seesaw contribution $m_{\nu}^{I}=-M_{D}^{\nu} M_{R}^{-1} M_{D}^{\nu^{T}}$ is exactly canceled out in the complete diagonalization method. Since light neutrino mass formula is independent of Dirac neutrino mass matrix, any value of $M_{D}^{\nu}$ is allowed consistent with GUT mass fitting without any fine tuning of the Yukawa couplings.

\subsection{Discussion on Neutrino Mass}

The relevant leptonic Yukawa interaction terms for extended seesaw mechanism are given by

$$
\begin{aligned}
-\mathcal{L}_{Y u k}= & \overline{\ell_{L}}\left[Y_{3} \Phi+Y_{4} \widetilde{\Phi}\right] \ell_{R}+f\left[\overline{\left(\ell_{L}\right)^{c}} \ell_{L} \Delta_{L}+\overline{\left(\ell_{R}\right)^{c}} \ell_{R} \Delta_{R}\right] \\
& +F \overline{\left(\ell_{R}\right)} H_{R} S_{L}^{c}+F^{\prime} \overline{\left(\ell_{L}\right)} H_{L} S_{L}+\mu_{S} \overline{S_{L}^{c}} S_{L}+\text { h.c. } \\
\supset & M_{D} \overline{\nu_{L}} N_{R}+M_{L} \overline{\nu_{L}^{c}} \nu_{L}+M_{R} \overline{N_{R}^{c}} N_{R} \\
& +M \overline{N_{R}} S_{L}+\mu_{L} \overline{\nu_{L}^{c}} S_{L}+\mu_{S} \overline{S_{L}^{c}} S_{L}
\end{aligned}
$$


After spontaneous symmetry breaking, the resulting neutral lepton mass matrix for extended seesaw mechanism in the basis $\left(\nu_{L}, N_{R}^{c}, S_{L}\right)$ is given by

$$
\mathbb{M}_{\nu}=\left(\begin{array}{ccc}
M_{L} & M_{D} & \mu_{L} \\
M_{D}^{T} & M_{R} & M^{T} \\
\mu_{L}^{T} & M & \mu_{S}
\end{array}\right)
$$

With $\left\langle H_{L}\right\rangle \rightarrow 0$ and $\mu_{S} \rightarrow 0$, the complete $9 \times 9$ neutral fermion mass matrix in the flavor basis of $\left(\nu_{L}, S_{L}, N_{R}^{c}\right)$ is read as

$$
\mathbb{M}=\left(\begin{array}{c|ccc} 
& \nu_{L} & S_{L} & N_{R}^{c} \\
\hline \nu_{L} & M_{L} & 0 & M_{D} \\
S_{L} & 0 & 0 & M \\
N_{R}^{c} & M_{D}^{T} & M^{T} & M_{R}
\end{array}\right)
$$

Using standard formalism of seesaw mechanism and using mass hierarchy $M_{R}>M>$ $M_{D} \gg M_{L}$, we can integrate out the heaviest right-handed neutrinos as follows

$$
\begin{aligned}
\mathbb{M}^{\prime} & =\left(\begin{array}{cc}
M_{L} & 0 \\
0 & 0
\end{array}\right)-\left(\begin{array}{c}
M_{D} \\
M
\end{array}\right) M_{R}^{-1}\left(M_{D}^{T} M^{T}\right) \\
& =\left(\begin{array}{cc}
M_{L}-M_{D} M_{R}^{-1} M_{D}^{T} & -M_{D} M_{R}^{-1} M^{T} \\
M M_{R}^{-1} M_{D}^{T} & -M M_{R}^{-1} M^{T}
\end{array}\right) .
\end{aligned}
$$

The block diagonalized mass matrices for light left-handed neutrinos, heavy right-handed neutrinos and extra sterile neutrinos are

$$
\begin{aligned}
& m_{\nu}=M_{L}, \\
& M_{N} \equiv M_{R}=\frac{v_{R}}{v_{L}} M_{L}, \\
& M_{S}=-M M_{R}^{-1} M^{T} .
\end{aligned}
$$

These block diagonalized mass matrices can be further diagonalized by respective $3 \times 3$ unitarity matrices as follows

$$
\begin{aligned}
& m_{\nu}^{\text {diag }}=U_{\nu}^{\dagger} m_{\nu} U_{\nu}^{*}=\operatorname{diag} .\left(m_{1}, m_{2}, m_{3}\right) \\
& M_{S}^{\text {diag }}=U_{S}^{\dagger} M_{S} U_{S}^{*}=\operatorname{diag} .\left(M_{S_{1}}, M_{S_{2}}, M_{S_{3}}\right), \\
& M_{N}^{\text {diag }}=U_{N}^{\dagger} M_{N} U_{N}^{*}=\operatorname{diag} .\left(M_{N_{1}}, M_{N_{2}}, M_{N_{3}}\right) .
\end{aligned}
$$

Finally, the complete block diagonalization yields

$$
\begin{aligned}
\widehat{\mathbb{M}} & =\mathrm{V}_{9 \times 9}^{\dagger} \mathbb{M V}_{9 \times 9}^{*}=(\mathbb{W} \cdot \mathbb{U})^{\dagger} \mathbb{M}(\mathbb{W} \cdot \mathbb{U}) \\
& =\operatorname{diag} .\left(m_{1}, m_{2}, m_{3} ; M_{S_{1}}, M_{S_{2}}, M_{S_{3}} ; M_{N_{1}}, M_{N_{2}}, M_{N_{3}}\right)
\end{aligned}
$$

Here the block diagonalized mixing matrix $\mathbb{W}$ and the unitarity matrix $\mathbb{U}$. 


\subsection{Neutrinoless double beta decay from large light-heavy neutrino mixing}

We discuss here the new physics contributions to neutrinoless double beta decay due to large left-right neutrino mixing. In the present non-supersymmetric $S O(10)$ GUT where the $\mathrm{TeV}$ scale asymmetric left-right symmetry breaking is implemented with both Higgs doublets and triplets, the resulting light neutrino mass is governed by extended type-II seesaw mechanism. The type-II seesaw dominance not only provides mass relation between light and heavy neutrinos but also allows large Dirac neutrino mass and thereby gives large light-heavy neutrino mixing. This light-heavy neutrino mixing plays an important role in giving sizable contributions to neutrinoless double beta decay.

The process of neutrinoless double beta decay is governed by following charge current interaction for leptons and quarks as following way

$$
\begin{aligned}
& \mathcal{L}_{C C}^{\mathrm{q}}=\frac{g_{L}}{\sqrt{2}} \bar{d} \gamma^{\mu} P_{L} u W_{L \mu}^{-}+\frac{g_{R}}{\sqrt{2}} \bar{d} \gamma^{\mu} P_{R} u W_{R \mu}^{-}+\text {h.c. }, \\
& \mathcal{L}_{C C}^{\mathrm{lep}}=\frac{g_{L}}{\sqrt{2}} \sum_{\alpha=e, \mu, \tau} \bar{\ell}_{\alpha} \gamma^{\mu} P_{L} \nu_{\alpha} W_{L \mu}^{-}+\frac{g_{R}}{\sqrt{2}} \sum_{\alpha=e, \mu, \tau} \bar{\ell}_{\alpha} \gamma_{\mu} P_{R} N_{\alpha} W_{R \mu}^{-}+\text {h.c. }
\end{aligned}
$$

Here the light neutrinos with flavor $\alpha=e, \mu, \tau$ is related with mass eigenstates of neutral leptons as

$$
\nu_{\alpha}=U_{\alpha i} \nu_{i}+\Theta_{\alpha j} N_{j}+Y_{\alpha k} S_{k} .
$$

From the unification plot, it is clear that the mass of right-handed charge gauge boson $W_{R}$ is kept at very hight scale. Because of large value of $M_{W_{R}}$, the new physics contributions to $0 \nu \beta \beta$ due to purely right-handed current is negligible as these contributions are proportional to $1 / M_{W_{R}}^{4}$. Similarly the well known $\lambda-\left(\propto 1 / M_{W_{R}}^{2}\right)$ and $\eta-\left(\propto \tan \theta_{L R}^{W}\right)$ diagrams are very much suppressed. The only contributions which can contribute to $0 \nu \beta \beta$ are arising from purely left-handed currents due to exchange of light left-handed neutrinos $\nu_{L}$, right-handed neutrinos $N_{R}$ and sterile neutrinos $S_{L}$ as displayed in Fig. 5.
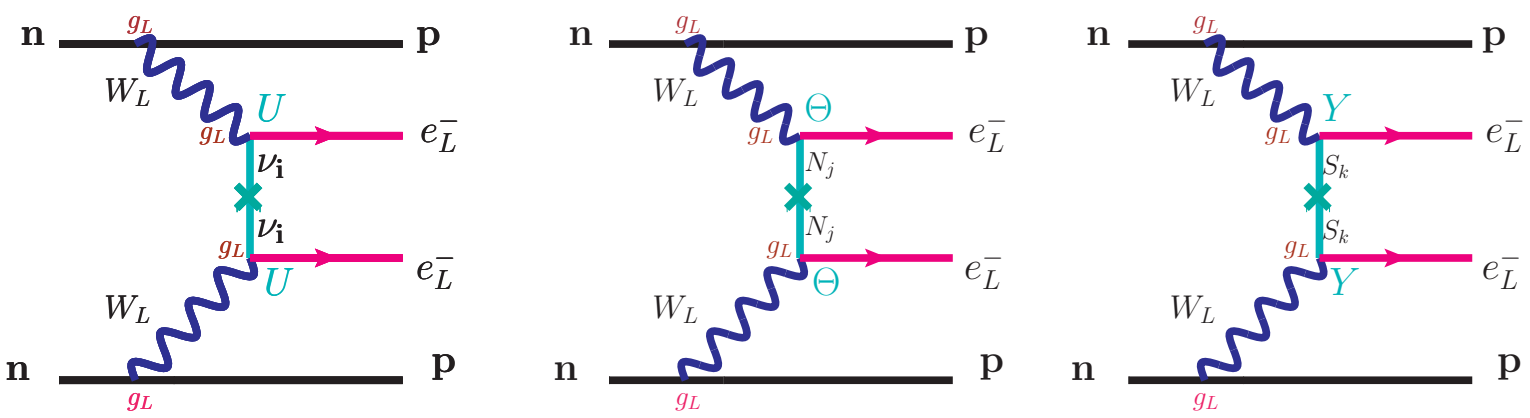

Figure 5. Feynman diagrams for neutrinoless double beta decay via $W_{L}^{-}-W_{L}^{-}$mediation with the exchange of virtual Majorana neutrinos $\nu_{i}, N_{j}$ and $S_{k}$.

Since the flavor neutrino state $\nu_{\alpha}$ is a linear combination of mass eigenstates $\nu_{i}, N_{j}, S_{k}$, there are three different Feynman diagrams contributing to neutrinoless double beta decay 


\begin{tabular}{c|c}
\hline \hline Effective Mass Parameters & Analytic formula \\
\hline $\mathbf{m}_{\mathrm{ee}, \mathrm{L}}^{\nu}$ & $\sum_{i=1}^{3} U_{e i}^{2} m_{\nu_{i}}$ \\
$\mathbf{m}_{\mathrm{ee}, \mathrm{L}}^{N}$ & $\sum_{i=1}^{3} \Theta_{e i}^{2} i|p|^{2}$ \\
$\mathbf{m}_{\mathrm{N}_{i}}$ & $\sum_{i=1}^{3} Y_{e i}^{2} \frac{|p|^{2}}{M_{S_{i}}}$ \\
\hline \hline
\end{tabular}

Table 4. The effective Majorana mass parameters arising from purely left-handed currents due to exchange of left-handed neutrinos $\nu_{L}$, right-handed neutrinos $N_{R}$ and sterile neutrinos $S_{L}$.

arising from purely left-handed charge current interaction as shown in Fig. 5. The Feynman amplitude for these diagrams are proportional to

$$
\begin{aligned}
& \mathcal{A}_{L L}^{\nu} \propto G_{F}^{2} \sum_{i=1,2,3} \frac{U_{e i}^{2} m_{\nu_{i}}}{p^{2}}, \\
& \mathcal{A}_{L L}^{N} \propto G_{F}^{2} \sum_{j=1,2,3}\left(-\frac{\Theta_{e j}^{2}}{M_{N_{j}}}\right), \\
& \mathcal{A}_{L L}^{S} \propto G_{F}^{2} \sum_{k=1,2,3}\left(-\frac{Y_{e k}^{2}}{M_{S_{k}}}\right),
\end{aligned}
$$

The resulting effective Majorana mass parameters, a measure of lepton number violation for these contributions are given by

$$
\left|\left\langle m_{e e}\right\rangle^{\nu}\right|=\sum_{i=1,2,3} U_{e i}^{2} m_{\nu_{i}}, \quad\left|\left\langle m_{e e}\right\rangle^{N}\right|=\left\langle p^{2}\right\rangle \sum_{j=1,2,3} \frac{\Theta_{e j}^{2}}{M_{N_{j}}}, \quad\left|\left\langle m_{e e}\right\rangle^{S}\right|=\left\langle p^{2}\right\rangle \sum_{k=1,2,3} \frac{Y_{e k}^{2}}{M_{S_{k}}} .
$$

The corresponding inverse half-life expression for this rare process $0 \nu \beta \beta$ due to exchange of $\nu, N$ and $S$ is given as follows

$$
\left[T_{1 / 2}^{0 \nu}\right]^{-1}=\mathcal{K}_{0 \nu}\left\{\left|\mathbf{m}_{\mathrm{ee}}^{\nu}\right|^{2}+\left|\mathbf{m}_{\mathrm{ee}}^{S}+\mathbf{m}_{\mathrm{ee}}^{N}\right|^{2}\right\}
$$

where $\mathcal{K}_{0 \nu} \simeq 1.57 \times 10^{-25} \mathrm{yrs}^{-1} \mathrm{eV}^{-2}$ is the product of the phase space factor, square of the nuclear matrix elements (NME) and the inverse of electron mass square.

\subsection{Numerical result for $0 \nu \beta \beta$}

We aim to present numerically different new physics contributions to $0 \nu \beta \beta$ transition with the variation of lightest neutrino mass and derive lower bound on lightest neutrino mass by saturating the existing experimental bound on $0 \nu \beta \beta$. The input model parameters needed for the said purpose are listed as follows:

\section{- Structure of $M_{D}$}

Since $S O(10)$ GUT contains Pati-Salam symmetry as highest subgroup relating quarks and leptons, the structure of Dirac neutrino mass matrix is expected to be of the order of up-type quark mass matrix. Assuming TeV scale asymmetric left-right symmetry 
originated from Pati-Salam symmetry [12] or $S O(10)$ GUT [136], and without considering RG corrections, the form of $M_{D}$ is derived to be ${ }^{1}$

$$
\begin{aligned}
M_{D} & =V_{C K M} M_{u} V_{C K M}^{T} \\
& =\left(\begin{array}{ccc}
0.067-0.004 i & 0.302-0.022 i & 0.550-0.530 i \\
0.302-0.022 i & 1.480 & 6.534-0.001 i \\
0.550-0.530 i & 6.534-0.0009 i & 159.72
\end{array}\right) \mathrm{GeV},
\end{aligned}
$$

where the PDG [140] value of up-type quark mass matrix and the CKM mixing matrix are given as follows

$$
\begin{aligned}
& M_{u}=\operatorname{diag}(2.3 \mathrm{MeV}, 1.275 \mathrm{GeV}, 173.210 \mathrm{GeV}), \\
& V_{C K M}=\left(\begin{array}{ccc}
0.97427 & 0.22534 & 0.00351-i 0.0033 \\
-0.2252+i 0.0001 & 0.97344 & 0.0412 \\
0.00876-i 0.0032 & -0.0404-i 0.0007 & 0.99912
\end{array}\right)
\end{aligned}
$$

\section{- Structure of $M$}

The $N_{R}-S_{L}$ mixing matrix $M$ is assumed to be diagonal and degenerate for simplicity of the numerical calculations and the mass matrix is given by

$$
M=m_{0}\left(\begin{array}{lll}
1 & 0 & 0 \\
0 & 1 & 0 \\
0 & 0 & 1
\end{array}\right),
$$

where we chose $m_{0}$ to be $200 \mathrm{GeV}, 500 \mathrm{GeV}$ and $1000 \mathrm{GeV}$ for our numerical estimations.

\section{- Physical masses and mixing for neutral leptons}

The complete diagonalization of neutral lepton in LRSM with additional fermion singlet has been discussed in Ref. [141] and we summarise them for our present discussion. The physical masses for light left-handed Majorana neutrinos, heavy righthanded Majorana neutrinos and sterile neutrinos in terms of oscillation parameters is given by

$$
\begin{aligned}
& m_{\nu}=U_{\mathrm{PMNS}} m_{\nu}^{\text {diag }} U_{\mathrm{PMNS}}^{T} \\
& M_{N} \equiv M_{R}=\frac{v_{R}}{v_{L}} U_{\mathrm{PMNS}} m_{\nu}^{\mathrm{diag}} U_{\mathrm{PMNS}}^{T} \\
& M_{S}=-m_{0}^{2} \frac{v_{L}}{v_{R}} U_{\mathrm{PMNS}}^{*} m_{\nu}^{\mathrm{diag}}{ }^{-1} U_{\mathrm{PMNS}}^{\dagger}
\end{aligned}
$$

The relevant mixing elements needed for $0 \nu \beta \beta$ are like light-light active neutrino mixing matrix as $U_{\nu}=U_{\text {PMNS }}$, mixing between left-handed and right-handed neutrinos as $\Theta=\frac{v_{L}}{v_{R}} M_{D} U_{\text {PMNS }}^{-1} m_{\nu}^{\text {diag }^{-1}}$ and mixing element between light active neutrinos and sterile neutrinos as $Y=\frac{1}{m_{0}} M_{D} U_{\mathrm{PMNS}}^{*}$.

\footnotetext{
${ }^{1}$ One can go through refs. [73, 137-139] where RG effect has been taken into account in constructing the Dirac neutrino mass matrix at $\mathrm{TeV}$ scale.
} 


\section{- $U_{\mathrm{PMNS}}$ and Oscillation Parameters}

The light active neutrino matrix can be diagonalized by the Pontecorvo-Maki-NakagawaSakata (PMNS) mixing matrix $U_{\text {PMNS }}$ as

$$
m_{\nu}^{\text {diag }}=U_{\mathrm{PMNS}}^{\dagger} m_{\nu} U_{\mathrm{PMNS}}^{*}=\operatorname{diag} .\left(m_{1}, m_{2}, m_{3}\right)
$$

where

$$
U_{\mathrm{PMNS}}=\left(\begin{array}{ccc}
c_{13} c_{12} & c_{13} s_{12} & s_{13} e^{-i \delta} \\
-c_{23} s_{12}-c_{12} s_{13} s_{23} e^{i \delta} & c_{12} c_{23}-s_{12} s_{13} s_{23} e^{i \delta} & s_{23} c_{13} \\
s_{12} s_{23}-c_{12} c_{23} s_{13} e^{i \delta} & -c_{12} s_{23}-s_{12} s_{13} c_{23} e^{i \delta} & c_{13} c_{23}
\end{array}\right) \cdot \mathrm{P} \cdot(5.21)
$$

We denote $s_{i j}=\sin \theta_{i j}, c_{i j}=\cos \theta_{i j}$ and diagonal phase matrix $\mathrm{P}=\operatorname{diag}\left(1, e^{i \alpha}, e^{i \beta}\right)$, where $\delta$ is the Dirac CP phase and $\alpha, \beta$ are Majorana phases. We vary them from 0 to $2 \pi$ in our numerical computation. The mixing angles like the atmospheric mixing angle as $\theta_{a} \equiv \theta_{23}$, the solar mixing angle as $\theta_{s} \equiv \theta_{12}$, and the reactor mixing angle as $\theta_{r} \equiv \theta_{13}$ and their $3 \sigma$ ranges are presented in Table. 5 along with the two mass squared differences like $\left(\Delta m_{\text {atm }}^{2}\right)$ and $\left(\Delta m_{\text {sol }}^{2}\right)$. Since oscillation experiments unable to provide precise measurement of the sign of $\Delta m_{\mathrm{atm}}^{2}$, there can be two possibilities in the arrangement of light neutrino masses like

Normal hierarchy (NH): $\Delta m_{\mathrm{atm}}^{2} \equiv \Delta m_{31}^{2}>0$, which gives $m_{1}<m_{2}<m_{3}$ with

$$
m_{2}=\sqrt{m_{1}^{2}+\Delta m_{\mathrm{sol}}^{2}}, \quad m_{3}=\sqrt{m_{1}^{2}+\Delta m_{\mathrm{atm}}^{2}},
$$

Inverted hierarchy (IH): $\Delta m_{\mathrm{atm}}^{2} \equiv \Delta m_{31}^{2}<0$, implying $m_{3}<m_{1}<m_{2}$ with

$$
m_{1}=\sqrt{m_{3}^{2}+\Delta m_{\mathrm{atm}}^{2}}, \quad m_{2}=\sqrt{m_{3}^{2}+\Delta m_{\mathrm{atm}}^{2}+\Delta m_{\mathrm{sol}}^{2}} .
$$

\begin{tabular}{cccc}
\hline Oscillation Parameters & $\begin{array}{c}3 \sigma \text { range } \\
\text { (Schwetz et al.[142]) }\end{array}$ & $\begin{array}{c}3 \sigma \text { range } \\
(\text { Fogli et al.[143]) }\end{array}$ & $\begin{array}{c}3 \sigma \text { range } \\
\text { (Gonzalez-Garcia et al [144]) }\end{array}$ \\
\hline \hline$\Delta m_{21}^{2}\left[10^{-5} \mathrm{eV}^{2}\right]$ & $7.00-8.09$ & $6.99-8.18$ & $7.02-8.09$ \\
$\left|\Delta m_{31}^{2}(\mathrm{NH})\right|\left[10^{-3} \mathrm{eV}^{2}\right]$ & $2.27-2.69$ & $2.19-2.62$ & $2.317-2.607$ \\
$\left|\Delta m_{31}^{2}(\mathrm{IH})\right|\left[10^{-3} \mathrm{eV}^{2}\right]$ & $2.24-2.65$ & $2.17-2.61$ & $2.307-2.590$ \\
\hline $\sin ^{2} \theta_{s}$ & $0.27-0.34$ & $0.259-0.359$ & $0.270-0.344$ \\
$\sin ^{2} \theta_{a}$ & $0.34-0.67$ & $0.331-0.637$ & $0.382-0.643$ \\
$\sin ^{2} \theta_{r}$ & $0.016-0.030$ & $0.017-0.031$ & $0.0186-0.0250$ \\
\hline
\end{tabular}

Table 5. The mass squared differences and mixing angles with their allowed $3 \sigma$ range. However we have taken the values given in Ref.[144] for our numerical computation.

In this work where the light neutrino mass is governed by type-II seesaw dominance, the heavy neutrino mass eigenvalues are related to light neutrino mass eigenvalues as follows: 
For NH pattern

$$
M_{N_{1}}=M_{1}=\frac{m_{1}}{m_{3}} M_{3}, \quad M_{N_{2}}=M_{2}=\frac{m_{2}}{m_{3}} M_{3} .
$$

where $M_{3}$ being the largest mass eigenvalue for right-handed Majorana neutrinos.

For IH pattern

$$
M_{N_{1}}=M_{1}=\frac{m_{1}}{m_{2}} M_{2}, \quad M_{N_{3}}=M_{3}=\frac{m_{3}}{m_{2}} M_{2} .
$$

$M_{2}$ being largest mass eigenvalue of right-handed neutrinos. We have fixed $M_{3}\left(M_{2}\right)$ as $5 \mathrm{TeV}$ for $\mathrm{NH}(\mathrm{IH})$ pattern.

The expression for inverse half-life for a given isotope for these contributions due to exchange of light neutrinos, heavy right-handed neutrinos and heavy sterile neutrinos is given by

$$
\left[T_{1 / 2}^{0 \nu}\right]^{-1}=G_{01}\left(\left|\mathcal{M}_{\nu} \eta_{\nu}\right|^{2}+\left|\mathcal{M}_{N}\left(\eta_{N}+\eta_{S}\right)\right|^{2}\right),
$$

where $G_{01}$ represents standard $0 \nu \beta \beta$ phase space factor, $\mathcal{M}_{i}$ as the corresponding nuclear matrix elements for the different exchange processes and $\eta_{i}$ are dimensionless particle physics parameters.

Due to Light neutrinos: The lepton number violating dimensionless particle physics parameter for $0 \nu \beta \beta$ transition due to the exchange of light neutrinos is given by

$$
\eta_{\nu}=\frac{1}{m_{e}} \sum_{i=1}^{3} U_{e i}^{2} m_{i}=\frac{m_{\mathrm{ee}}^{\nu}}{m_{e}},
$$

where $m_{e}$ is the electron mass.
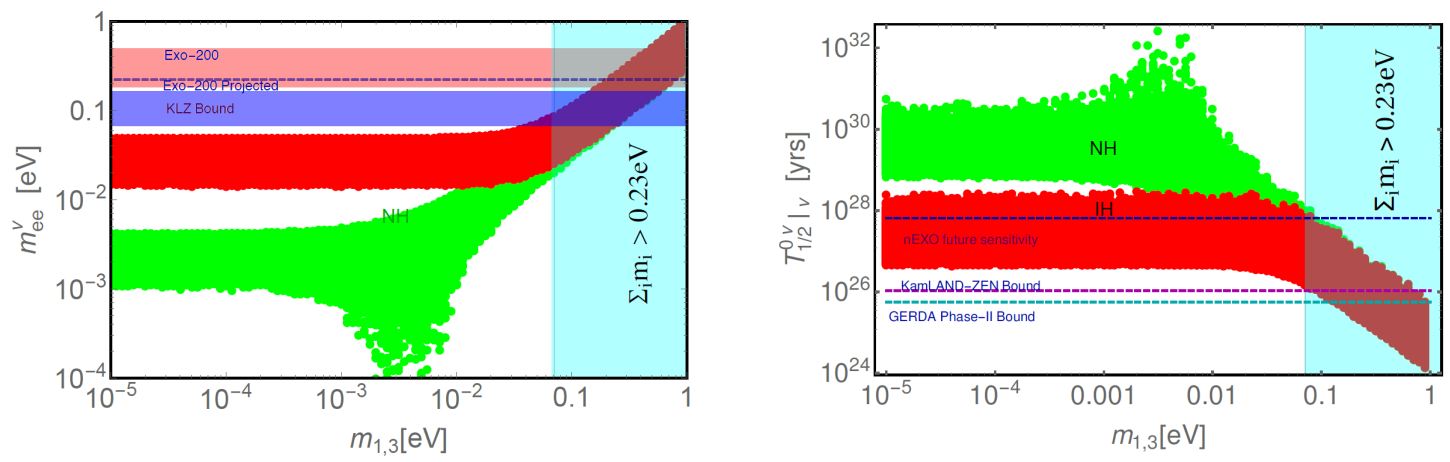

Figure 6. Effective Majorana mass parameter and Half-life of $0 \nu \beta \beta$ decay due to exchange of light active neutrinos as a function of the lightest neutrino mass for a $\mathrm{NH}$ and $\mathrm{IH}$ pattern.

The corresponding effective mass parameter is given by

$$
m_{\mathrm{ee}}^{\nu}=\left|c_{12}^{2} c_{13}^{2} m_{1}+s_{12}^{2} c_{13}^{2} m_{2} e^{i \alpha}+s_{13}^{2} m_{3} e^{i \beta}\right|,
$$

with $\theta_{12}$ and $\theta_{13}, c_{12}=\cos \theta_{12}$, etc as the respective sine and cosine of the oscillation angles. The two unconstrained Majorana phases varied between $0 \leq \alpha, \beta<2 \pi$. 

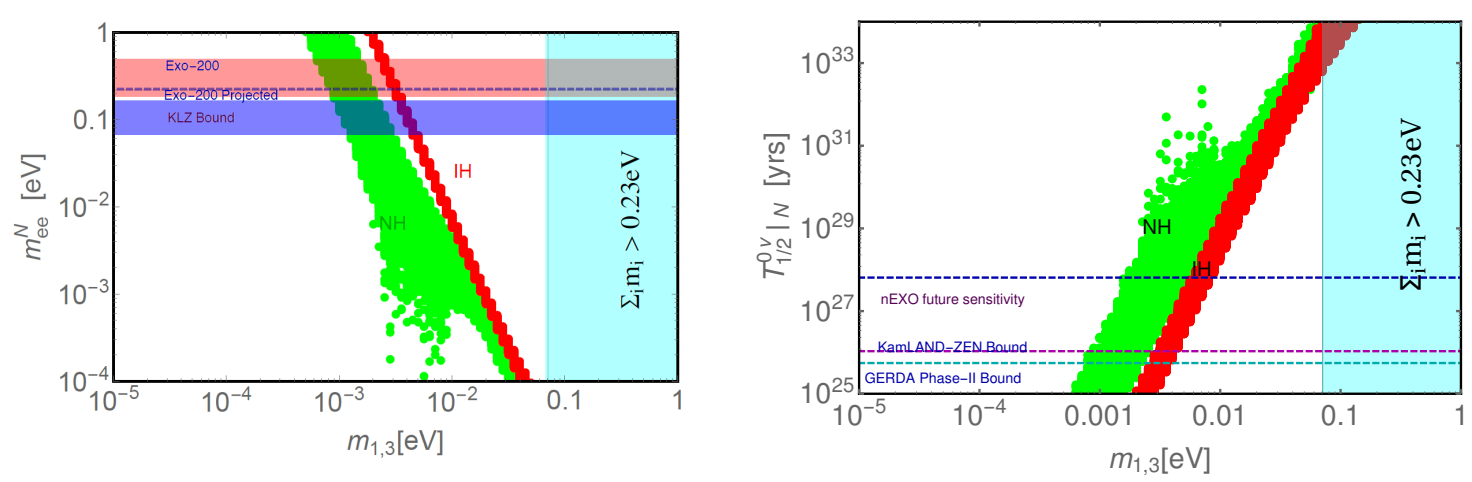

Figure 7. Effective Majorana mass parameter and Half-life of $0 \nu \beta \beta$ decay due to exchange of heavy right-handed neutrinos as a function of the lightest neutrino mass for a NH and IH pattern.

Due to right-handed neutrinos: The new non-standard contribution to $0 \nu \beta \beta$ decay arising from the purely left-handed currents via the exchange of right-handed neutrinos results in the lepton number violating dimensionless particle physics parameter

$$
\eta_{N}=m_{p} \sum_{i=1}^{3} \frac{\Theta_{e i}^{2} M_{i}}{|p|^{2}+M_{i}^{2}} .
$$

We define here $p$ as the virtual neutrino momentum of the order of the nuclear Fermi scale, $p \approx 100 \mathrm{MeV}$ and $m_{p}$ is the proton mass.

We also consider mass of right-handed neutrinos larger than nuclear fermi scale i.e, $M_{i}^{2} \gg\left|p^{2}\right|$. With $M_{i} \gg|p|$, the propagator simplifies as

$$
\frac{M_{i}}{p^{2}-M_{i}^{2}} \approx-\frac{1}{M_{i}}
$$

Thus, the effective dimensionless particle physics parameter due to exchange of heavy righthanded neutrinos is found to be

$$
\eta_{N}=-m_{p} \sum_{i=1}^{3} \frac{\Theta_{e i}^{2}}{M_{i}},
$$

and the corresponding effective Majorana mass parameter is given by

$$
m_{\mathrm{ee}}^{N}=\sum_{i=1}^{3} \frac{\Theta_{e i}^{2}}{M_{i}}\left\langle p^{2}\right\rangle .
$$

Due to heavy sterile neutrinos: Similarly, the contribution to $0 \nu \beta \beta$ decay arising from heavy sterile neutrinos gives lepton number violating dimensionless particle physics parameter as,

$$
\eta_{S}=m_{p} \sum_{i=1}^{3} \frac{Y_{e i}^{2} M_{S_{i}}}{|p|^{2}+M_{S_{i}}^{2}} .
$$



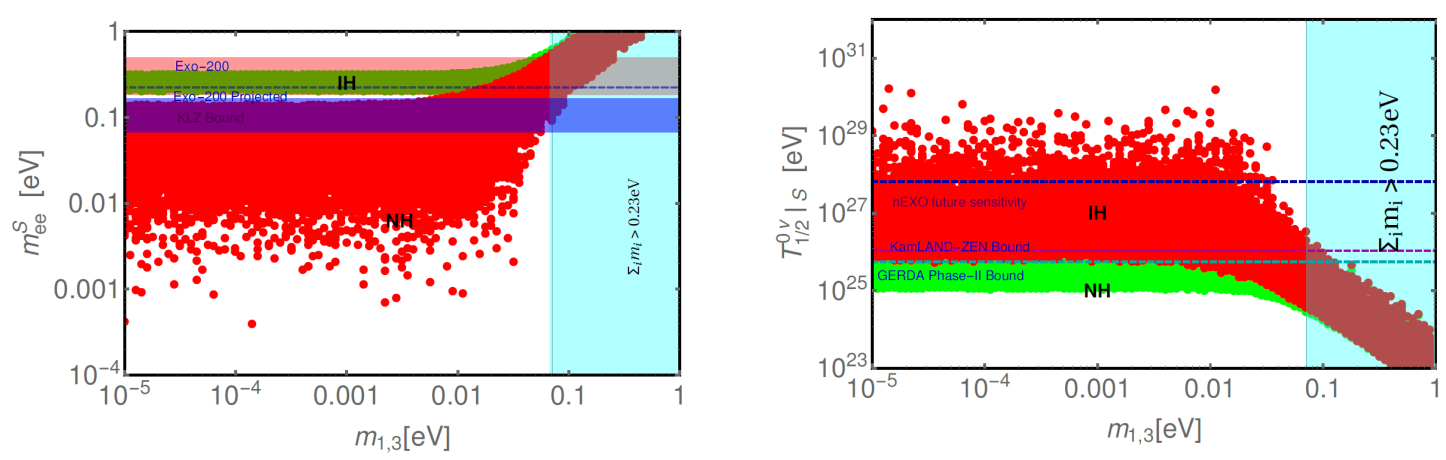

Figure 8. Effective Majorana mass parameter and Half-life of $0 \nu \beta \beta$ decay due to exchange of heavy sterile neutrinos as a function of the lightest neutrino mass for a NH and IH pattern.

Assuming $M_{S_{i}} \gg|p|$, the dimensionless particle physics parameter and effective Majorana mass parameter due to exchange of sterile neutrinos are expressed as,

$$
\eta_{S}=-m_{p} \sum_{i=1}^{3} \frac{Y_{e i}^{2}}{M_{S_{i}}}, \quad m_{\mathrm{ee}}^{S}=\sum_{i=1}^{3} \frac{Y_{e i}^{2}}{M_{S_{i}}}\left\langle p^{2}\right\rangle .
$$
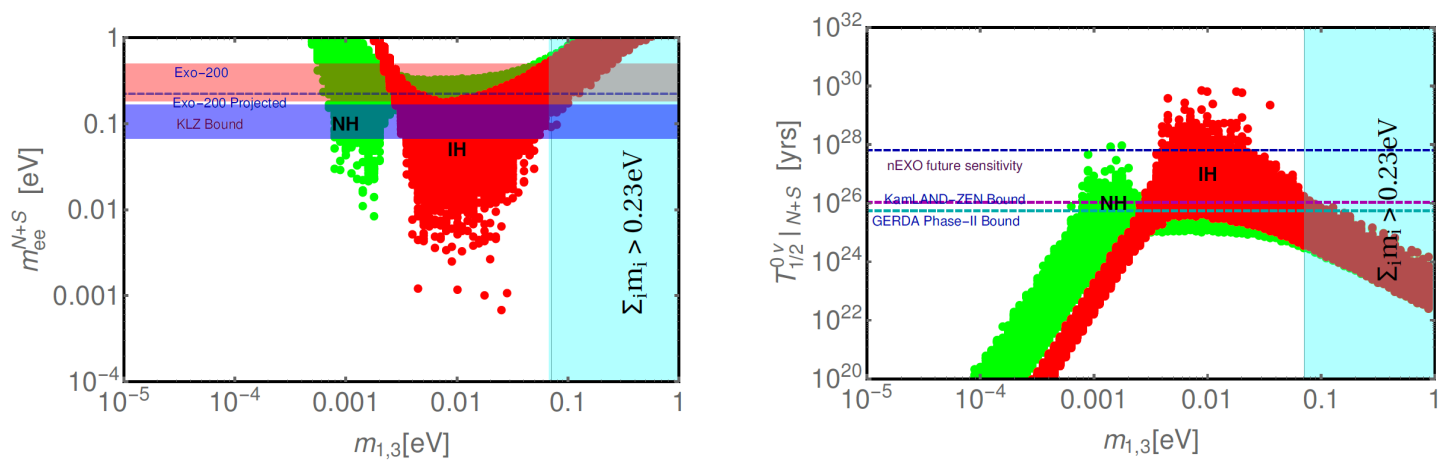

Figure 9. Effective Majorana mass parameter and Half-life of $0 \nu \beta \beta$ decay due to combine effect of heavy right-handed neutrinos plus sterile neutrinos as a function of the lightest neutrino mass for a $\mathrm{NH}$ and $\mathrm{IH}$ pattern.

Combined contributions The combined contribution to effective Majorana mass parameter due to exchange of heavy right-handed neutrinos as well as sterile neutrinos is given by

$$
m_{\mathrm{ee}}^{N+S}=\sum_{i=1}^{3} \frac{\Theta_{e i}^{2}}{M_{i}}\left\langle p^{2}\right\rangle+\sum_{i=1}^{3} \frac{Y_{e i}^{2}}{M_{S_{i}}}\left\langle p^{2}\right\rangle .
$$

Analogously one can write the combined contribution to half-life for $0 \nu \beta \beta$ decay due to exchange of light left-handed neutrinos, heavy right-handed neutrinos and sterile neutrinos 
is given by

$$
\begin{aligned}
{\left[T_{1 / 2}^{0 \nu}\right]^{-1} } & =G_{01}\left(\left|\mathcal{M}_{\nu} \eta_{\nu}\right|^{2}+\left|\mathcal{M}_{N}\left(\eta_{N}+\eta_{S}\right)\right|^{2}\right) \\
& =G_{01}\left|\frac{\mathcal{M}_{\nu}}{m_{e}}\right|^{2} \cdot\left|m_{\mathrm{ee}}^{\text {Tot }}\right|^{2}
\end{aligned}
$$

where the total contributions to effective mass parameter is found to be

$$
\left|m_{\mathrm{ee}}^{\mathrm{Tot}}\right|^{2}=\left|\sum_{i=1}^{3} U_{e i}^{2} m_{i}\right|^{2}+\left|\sum_{i=1}^{3} \frac{\Theta_{e i}^{2}}{M_{i}}\left\langle p^{2}\right\rangle\right|^{2}+\left|\sum_{i=1}^{3} \frac{Y_{e i}^{2}}{M_{S_{i}}}\left\langle p^{2}\right\rangle\right|^{2} .
$$

Using these input parameters and the $3 \sigma$ ranges of the oscillation data from Table-5, we show the variation of effective mass (left panel) and half-life (right panel) vs. lightest neutrino mass $m_{\text {lightest }}=m_{1}(\mathrm{NH}), m_{3}(\mathrm{IH})$ in Fig.-6 due to the exchange of light Majorana neutrino. The green (red) region represents the contributions from light neutrinos obeying Normal (Inverted) mass ordering. The cyan region is excluded due to the 95\% CL limit $\sum_{i} m_{i}<0.23 \mathrm{eV}$ obtained from Planck + WMAP low multipole polarization + high resolution $\mathrm{CMB}+\mathrm{BAO}$ data. The horizontal bands/lines on the left (right) plot represent the upper (lower) limit on $m_{e e}\left(T_{1 / 2}^{0 \nu}\right)$ from various experiments. From these figures it can be noted that if only the light Majorana neutrinos contribute to the $o \nu \beta \beta$ decay process, it is very difficult to see the signal even at next generation experiments. On the other hand if the new physics contributions arising from the exchange of right-handed or sterile neutrinos are included, the $m_{e e}$ and $T_{1 / 2}^{0 \nu}$ values are significantly enhanced as seen from Figs. 7 and 8 . The combined effects can saturate the current experimental limits as shown is Figs. 9 and 10, which in turn give the lower bound on the lightest neutrino mass as $m_{1}\left(m_{3}\right) \geq 1$ (5) $\mathrm{meV}$ for $\mathrm{NH}(\mathrm{IH})$.
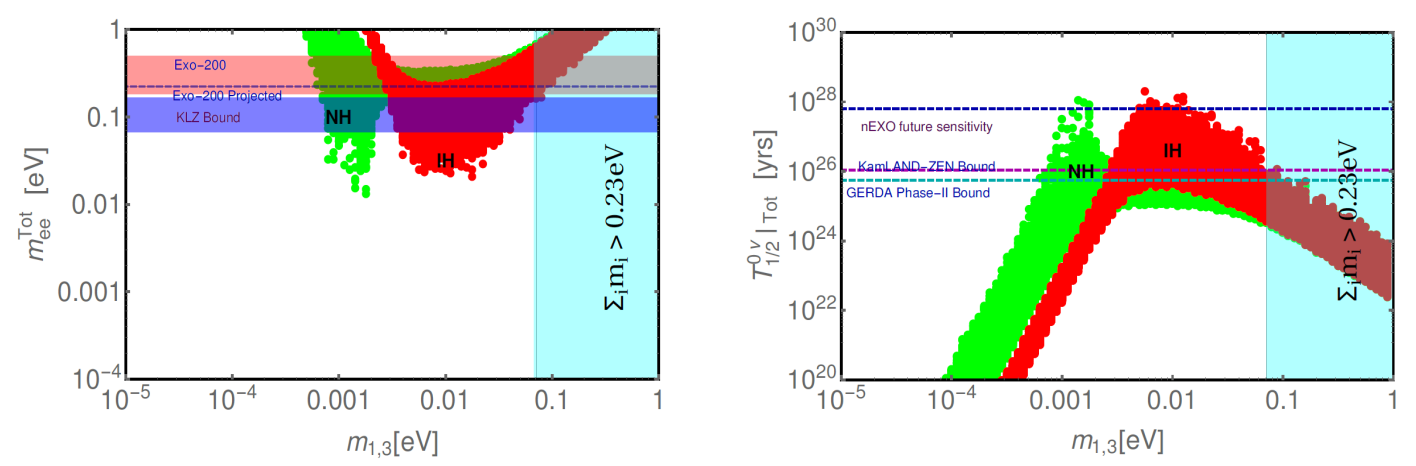

Figure 10. Effective Majorana mass parameter and Half-life of $0 \nu \beta \beta$ decay due to combine effect of light active left-handed neutrinos, heavy right-handed neutrinos and sterile neutrinos as a function of the lightest neutrino mass for a NH and IH pattern. 


\section{Conclusion}

In this work, we have investigated gauge coupling unification within a non-supersymmetric $S O(10)$ GUT with Pati-Salam symmetry and TeV scale asymmetric left-right theory as subgroups of the model. We have studied the RG evolution of gauge couplings for three different cases where the spontaneous symmetry breaking of asymmetric left-right symmetry is implemented with either Higgs doublets or triplets or combination of both. We found that, in the first case where the intermediate symmetry $U(1)_{R} \times U(1)_{B-L} \rightarrow U(1)_{Y}$ is broken via the Higgs doublet $\left(H_{R}^{0}\right)$ around $\mathrm{TeV}$ scale, the unification of gauge couplings occurs at the scale $M_{U} \sim 10^{16} \mathrm{GeV}$. The Pati-Salam symmetry breaks at $\mathcal{O}\left(10^{8}\right) \mathrm{GeV}$, around which the right handed gauge boson $W_{R}$ gets its mass. The light neutrino masses are predominantly Dirac type and their sub-eV scale can be achieved by suitable adjustment of different Yukawa couplings or by fine tuning of the parameters. Since there is no Majorana masses for the neutrinos, there will be no lepton number violation in this model. In the second case, the intermediate $U(1)_{R} \times U(1)_{B-L} \rightarrow U(1)_{Y}$ symmetry breaking is achieved through the Higgs triplets $\left(\Delta_{L, R}\right)$, the unification occurs at $\sim 10^{16} \mathrm{GeV}$ and the D-parity breaking scale is around $\sim 10^{12} \mathrm{GeV}$. Because of the lower D-parity breaking scale there is sub-dominant Type-II seesaw contribution to the light neutrino mass along with the dominant type-II seesaw contribution with $\mathrm{TeV}$ scale right-handed neutrinos. Also, the $S U(2)_{R}$ breaking occurs at a lower scale $\left(\sim 10^{5} \mathrm{GeV}\right)$, which allows right handed currents. The inclusion of Higgs doublets and triplets for the intermediate $U(1)_{R} \times U(1)_{B-L} \rightarrow U(1)_{Y}$ symmetry breaking yields almost similar results. With the asymmetric left-right symmetry breaking done with both combination of doublets and triplets, we have introduced one extra triplet to lower down the scale of D-parity breaking scale around $10^{10}-10^{11} \mathrm{GeV}$ so that the typeII seesaw contribution to light neutrino mass i.e, $m_{\nu}^{I I}=f v_{L} \approx \mathcal{O}(1) \cdot v^{2} \cdot v_{R} /\left(M^{\prime} \cdot M_{D_{P}}\right)$ is dominant.

We have also carried out a detail analysis on neutrino mass and neutrinoless double beta decay for the case where $\mathrm{TeV}$ scale asymmetric left-right symmetry breaking is done with both scalar doublets and triplets while adding an extra fermion singlet per generation to the minimal particle content. The resulting light neutrino mass formula is governed by natural type-II seesaw mechanism where mass eigenvalues for light and heavy neutrinos are related. The type-I seesaw contribution is exactly canceled out in the diagonalization of complete neutral lepton mass matrices and thus, there is no constraint on Dirac neutrino mass from light neutrino mass formula. We have considered Dirac neutrino mass matrix is equal to up-type quark mass matrix for all our numerical analysis which is a characteristics of Pati-Salam symmetry. It is well-known that $0 \nu \beta \beta$ process violate lepton number by two units and are mediated by Majorana neutrino mass terms which eventually manifest the Majorana nature of neutrinos. We found that if only the standard light active neutrinos are considered than the effective electron mass parameter $m_{e e}$ is few order magnitude smaller that the current limit of the next generation experiments and it will be very difficult to get a signal. Also we have shown that inclusion of the new physics contributions to neutrinoless double beta decay induced by the exchange of right-handed neutrinos and sterile neutrinos can saturate the present experimental limit and it is possible to see a signal at the next 
generation experiments. We also found the lower limits on the absolute mass scale of the lightest neutrinos $m_{1} \geq 1 \mathrm{meV}$ for $\mathrm{NH}$ and $m_{3} \geq 5 \mathrm{meV}$ for $\mathrm{IH}$.

\section{Acknowledgement}

SM would like to thank University Grants Commission for financial support. RM acknowledges the support from the Science and Engineering Research Board (SERB), Government of India through grant No. SB/S2/HEP-017/2013. SP would like to acknowledge the warm hospitality provided by University of Hyderabad, India, between $22^{\text {nd }}-29^{\text {th }}$ March, 2017, during which the part of this work is completed.

\section{References}

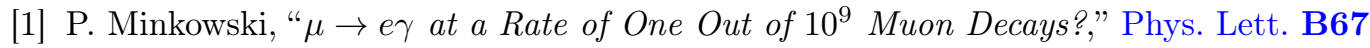
(1977) 421-428.

[2] R. N. Mohapatra and G. Senjanović, "Neutrino Mass and Spontaneous Parity Violation," Phys.Rev.Lett. 44 (1980) 912.

[3] T. Yanagida, "HORIZONTAL SYMMETRY AND MASSES OF NEUTRINOS," Conf. Proc. C7902131 (1979) 95-99.

[4] M. Gell-Mann, P. Ramond, and R. Slansky, "Complex Spinors and Unified Theories," Conf. Proc. C790927 (1979) 315-321, arXiv:1306.4669.

[5] J. Schechter and J. W. F. Valle, "Neutrino Masses in SU(2) x U(1) Theories," Phys. Rev. D22 (1980) 2227.

[6] M. Magg and C. Wetterich, "Neutrino Mass Problem and Gauge Hierarchy," Phys. Lett. B94 (1980) 61-64.

[7] T. P. Cheng and L.-F. Li, "Neutrino Masses, Mixings and Oscillations in SU(2) $x$ U(1) Models of Electroweak Interactions," Phys. Rev. D22 (1980) 2860.

[8] G. Lazarides, Q. Shafi, and C. Wetterich, "Proton Lifetime and Fermion Masses in an SO(10) Model," Nucl. Phys. B181 (1981) 287-300.

[9] R. N. Mohapatra and G. Senjanović, "Neutrino Masses and Mixings in Gauge Models with Spontaneous Parity Violation," Phys.Rev. D23 (1981) 165.

[10] C. Wetterich, "Neutrino Masses and the Scale of B-L Violation," Nucl. Phys. B187 (1981) 343-375.

[11] S. Antusch and S. F. King, "Type II Leptogenesis and the neutrino mass scale," Phys. Lett. B597 (2004) 199-207, arXiv:hep-ph/0405093.

[12] J. C. Pati and A. Salam, "Lepton Number as the Fourth Color," Phys.Rev. D10 (1974) $275-289$.

[13] R. Mohapatra and J. C. Pati, "A Natural Left-Right Symmetry," Phys.Rev. D11 (1975) 2558.

[14] G. Senjanović and R. N. Mohapatra, "Exact Left-Right Symmetry and Spontaneous Violation of Parity," Phys.Rev. D12 (1975) 1502. 
[15] N. G. Deshpande, J. F. Gunion, B. Kayser, and F. I. Olness, "Left-right symmetric electroweak models with triplet Higgs," Phys. Rev. D44 (1991) 837-858.

[16] R. N. Mohapatra and R. E. Marshak, "Local B-L Symmetry of Electroweak Interactions, Majorana Neutrinos and Neutron Oscillations," Phys. Rev. Lett. 44 (1980) 1316-1319. [Erratum: Phys. Rev. Lett.44,1643(1980)].

[17] W.-Y. Keung and G. Senjanović, "Majorana Neutrinos and the Production of the Right-handed Charged Gauge Boson," Phys.Rev.Lett. 50 (1983) 1427.

[18] A. Ferrari, J. Collot, M.-L. Andrieux, B. Belhorma, P. de Saintignon, J.-Y. Hostachy, P. Martin, and M. Wielers, "Sensitivity study for new gauge bosons and right-handed Majorana neutrinos in pp collisions at $s=14-T e V$," Phys. Rev. D62 (2000) 013001.

[19] M. Schmaltz and C. Spethmann, "Two Simple W' Models for the Early LHC," JHEP 07 (2011) 046, arXiv:1011.5918.

[20] M. Nemevsek, F. Nesti, G. Senjanovic, and Y. Zhang, "First Limits on Left-Right Symmetry Scale from LHC Data," Phys. Rev. D83 (2011) 115014, arXiv:1103.1627.

[21] C.-Y. Chen and P. S. B. Dev, "Multi-Lepton Collider Signatures of Heavy Dirac and Majorana Neutrinos," Phys. Rev. D85 (2012) 093018, arXiv:1112.6419.

[22] J. Chakrabortty, J. Gluza, R. Sevillano, and R. Szafron, "Left-Right Symmetry at LHC and Precise 1-Loop Low Energy Data," JHEP 07 (2012) 038, arXiv:1204.0736.

[23] S. Das, F. Deppisch, O. Kittel, and J. Valle, "Heavy Neutrinos and Lepton Flavour Violation in Left-Right Symmetric Models at the LHC," Phys.Rev. D86 (2012) 055006, arXiv: 1206.0256.

[24] J. A. Aguilar-Saavedra and F. R. Joaquim, "Measuring heavy neutrino couplings at the LHC," Phys. Rev. D86 (2012) 073005, arXiv:1207.4193.

[25] T. Han, I. Lewis, R. Ruiz, and Z.-g. Si, "Lepton Number Violation and $W^{\prime}$ Chiral Couplings at the LHC," Phys. Rev. D87 (2013) no. 3, 035011, arXiv:1211.6447. [Erratum: Phys. Rev.D87,no.3,039906(2013)].

[26] C.-Y. Chen, P. S. B. Dev, and R. N. Mohapatra, "Probing Heavy-Light Neutrino Mixing in Left-Right Seesaw Models at the LHC," Phys. Rev. D88 (2013) 033014, arXiv:1306.2342.

[27] T. G. Rizzo, "Exploring new gauge bosons at a 100 TeV collider," Phys. Rev. D89 (2014) no. 9, 095022, arXiv:1403.5465.

[28] F. F. Deppisch, T. E. Gonzalo, S. Patra, N. Sahu, and U. Sarkar, "Double beta decay, lepton flavor violation, and collider signatures of left-right symmetric models with spontaneous D-parity breaking," Phys. Rev. D91 (2015) no. 1, 015018, arXiv:1410.6427.

[29] F. F. Deppisch, P. S. Bhupal Dev, and A. Pilaftsis, "Neutrinos and Collider Physics," New J. Phys. 17 (2015) no. 7, 075019, arXiv:1502.06541.

[30] J. Gluza and T. JeliÅĎski, "Heavy neutrinos and the $p p \rightarrow l l j j$ CMS data," Phys. Lett. B748 (2015) 125-131, arXiv:1504.05568.

[31] J. N. Ng, A. de la Puente, and B. W.-P. Pan, "Search for Heavy Right-Handed Neutrinos at the LHC and Beyond in the Same-Sign Same-Flavor Leptons Final State," JHEP 12 (2015) 172, arXiv: 1505.01934.

[32] S. Patra, F. S. Queiroz, and W. Rodejohann, "Stringent Dilepton Bounds on Left-Right Models using LHC data," Phys. Lett. B752 (2016) 186-190, arXiv:1506. 03456. 
[33] B. A. Dobrescu and Z. Liu, "WâĂ̌̌ Boson near 2 TeV: Predictions for Run 2 of the LHC," Phys. Rev. Lett. 115 (2015) no. 21, 211802, arXiv:1506.06736.

[34] J. Brehmer, J. Hewett, J. Kopp, T. Rizzo, and J. Tattersall, "Symmetry Restored in Dibosons at the LHC?," JHEP 10 (2015) 182, arXiv:1507.00013.

[35] P. S. Bhupal Dev and R. N. Mohapatra, "Unified explanation of the eejj, diboson and dijet resonances at the LHC," Phys. Rev. Lett. 115 (2015) no. 18, 181803, arXiv:1508.02277.

[36] P. Coloma, B. A. Dobrescu, and J. Lopez-Pavon, "Right-handed neutrinos and the $2 \mathrm{TeV}$ $W^{\prime}$ boson," Phys. Rev. D92 (2015) no. 11, 115023, arXiv:1508.04129.

[37] F. F. Deppisch, L. Graf, S. Kulkarni, S. Patra, W. Rodejohann, N. Sahu, and U. Sarkar, "Reconciling the $2 \mathrm{TeV}$ excesses at the LHC in a linear seesaw left-right model," Phys. Rev. D93 (2016) no. 1, 013011, arXiv:1508.05940.

[38] P. S. B. Dev, D. Kim, and R. N. Mohapatra, "Disambiguating Seesaw Models using Invariant Mass Variables at Hadron Colliders," JHEP 01 (2016) 118, arXiv:1510.04328.

[39] S. Mondal and S. K. Rai, "Polarized window for left-right symmetry and a right-handed neutrino at the Large Hadron-Electron Collider," Phys. Rev. D93 (2016) no. 1, 011702, arXiv: 1510.08632.

[40] J. A. Aguilar-Saavedra and F. R. Joaquim, "Multiboson production in $W^{\prime}$ decays," JHEP 01 (2016) 183, arXiv:1512.00396.

[41] M. Lindner, F. S. Queiroz, and W. Rodejohann, "Dilepton bounds on left-right symmetry at the LHC run II and neutrinoless double beta decay," arXiv:1604.07419.

[42] M. Lindner, F. S. Queiroz, W. Rodejohann, and C. E. Yaguna, "Left-Right Symmetry and Lepton Number Violation at the Large Hadron Electron Collider," JHEP 06 (2016) 140, arXiv: 1604.08596.

[43] M. Mitra, R. Ruiz, D. J. Scott, and M. Spannowsky, "Neutrino Jets from High-Mass $W_{R}$ Gauge Bosons in TeV-Scale Left-Right Symmetric Models," Phys. Rev. D94 (2016) no. 9, 095016, arXiv: 1607.03504.

[44] G. Anamiati, M. Hirsch, and E. Nardi, "Quasi-Dirac neutrinos at the LHC," JHEP 10 (2016) 010, arXiv: 1607.05641.

[45] CMS, V. Khachatryan et al., "Search for heavy neutrinos and W bosons with right-handed couplings in proton-proton collisions at $\sqrt{s}=8$ TeV," Eur.Phys.J. C74 (2014) 3149, arXiv: 1407.3683.

[46] ATLAS, G. Aad et al., "Search for heavy Majorana neutrinos with the ATLAS detector in pp collisions at $\sqrt{s}=8 \mathrm{TeV}$," JHEP 07 (2015) 162, arXiv:1506.06020.

[47] CMS, V. Khachatryan et al., "Search for heavy neutrinos or third-generation leptoquarks in final states with two hadronically decaying tau leptons and two jets in proton-proton collisions at sqrt(s) =13 TeV," arXiv:1612.01190.

[48] J. F. Gunion, J. Grifols, A. Mendez, B. Kayser, and F. I. Olness, "Higgs Bosons in Left-Right Symmetric Models," Phys. Rev. D40 (1989) 1546.

[49] J. Polak and M. Zralek, "Higgs sector influence on left-right symmetric model parameters," Phys. Lett. B276 (1992) 492-496.

[50] G. Barenboim, M. Gorbahn, U. Nierste, and M. Raidal, "Higgs sector of the minimal left-right symmetric model," Phys. Rev. D65 (2002) 095003, arXiv:hep-ph/0107121. 
[51] G. Azuelos, K. Benslama, and J. Ferland, "Prospects for the search for a doubly-charged Higgs in the left-right symmetric model with ATLAS," J. Phys. G32 (2006) no. 2, 73-91, arXiv: hep-ph/0503096.

[52] D.-W. Jung and K. Y. Lee, "Production of the charged Higgs bosons at the CERN Large Hadron Collider in the left-right symmetric model," Phys. Rev. D78 (2008) 015022, arXiv:0802.1572.

[53] G. Bambhaniya, J. Chakrabortty, J. Gluza, M. KordiaczyÅĎska, and R. Szafron, "Left-Right Symmetry and the Charged Higgs Bosons at the LHC," JHEP 05 (2014) 033, arXiv: 1311.4144 .

[54] B. Dutta, R. Eusebi, Y. Gao, T. Ghosh, and T. Kamon, "Exploring the doubly charged Higgs boson of the left-right symmetric model using vector boson fusionlike events at the LHC," Phys. Rev. D90 (2014) 055015, arXiv:1404.0685.

[55] G. Bambhaniya, J. Chakrabortty, J. Gluza, T. JeliÅĎski, and M. Kordiaczynska, "Lowest limits on the doubly charged Higgs boson masses in the minimal left-right symmetric model," Phys. Rev. D90 (2014) no. 9, 095003, arXiv:1408.0774.

[56] G. Bambhaniya, J. Chakrabortty, J. Gluza, T. Jelinski, and R. Szafron, "Search for doubly charged Higgs bosons through vector boson fusion at the LHC and beyond," Phys. Rev. D92 (2015) no. 1, 015016, arXiv:1504.03999.

[57] P. S. B. Dev, R. N. Mohapatra, and Y. Zhang, "Probing the Higgs Sector of the Minimal Left-Right Symmetric Model at Future Hadron Colliders," JHEP 05 (2016) 174, arXiv: 1602.05947.

[58] ATLAS, G. Aad et al., "Search for anomalous production of prompt same-sign lepton pairs and pair-produced doubly charged Higgs bosons with $\sqrt{s}=8 \mathrm{TeV}$ pp collisions using the ATLAS detector," JHEP 03 (2015) 041, arXiv:1412.0237.

[59] CMS, C. Collaboration, "Search for a doubly-charged Higgs boson with $\sqrt{s}=8 \mathrm{TeV} p p$ collisions at the CMS experiment,".

[60] ATLAS, T. A. collaboration, "Search for doubly-charged Higgs bosons in same-charge electron pair final states using proton-proton collisions at $\sqrt{s}=13 \mathrm{TeV}$ with the ATLAS detector,".

[61] C. Hati, S. Patra, M. Reig, J. W. F. Valle, and C. A. Vaquera-Araujo, "Towards gauge coupling unification in left-right symmetric $\mathrm{SU}(3)_{c} \times \mathrm{SU}(3)_{\mathrm{L}} \times \mathrm{SU}(3)_{\mathrm{R}} \times \mathrm{U}(1)_{\mathrm{X}}$ theories," arXiv: 1703.09647.

[62] D. Borah, S. Patra, and U. Sarkar, "TeV scale Left Right Symmetry with spontaneous D-parity breaking," Phys. Rev. D83 (2011) 035007, arXiv:1006.2245.

[63] R. Kuchimanchi, "Scale of left-right symmetry," arXiv:1704.07249.

[64] P. S. B. Dev, R. N. Mohapatra, and Y. Zhang, "Displaced Photon Signal from a Light Scalar in Minimal Left-Right Seesaw Model," arXiv:1612.09587.

[65] R. N. Mohapatra and J. D. Vergados, "A New Contribution to Neutrinoless Double Beta Decay in Gauge Models," Phys. Rev. Lett. 47 (1981) 1713-1716.

[66] C. E. Picciotto and M. S. Zahir, "Neutrinoless Double Beta Decay in Left-right Symmetric Models," Phys. Rev. D26 (1982) 2320. 
[67] M. Hirsch, H. V. Klapdor-Kleingrothaus, and O. Panella, "Double beta decay in left-right symmetric models," Phys. Lett. B374 (1996) 7-12, arXiv:hep-ph/9602306.

[68] SuperNEMO, R. Arnold et al., "Probing New Physics Models of Neutrinoless Double Beta Decay with SuperNEMO," Eur. Phys. J. C70 (2010) 927-943, arXiv:1005.1241.

[69] V. Tello, M. Nemevsek, F. Nesti, G. Senjanovic, and F. Vissani, "Left-Right Symmetry: from LHC to Neutrinoless Double Beta Decay," Phys. Rev. Lett. 106 (2011) 151801, arXiv: 1011.3522.

[70] J. Chakrabortty, H. Z. Devi, S. Goswami, and S. Patra, "Neutrinoless double- $\beta$ decay in TeV scale Left-Right symmetric models," JHEP 08 (2012) 008, arXiv:1204.2527.

[71] M. Nemevsek, G. Senjanovic, and V. Tello, "Connecting Dirac and Majorana Neutrino Mass Matrices in the Minimal Left-Right Symmetric Model," Phys. Rev. Lett. 110 (2013) no. 15, 151802, arXiv:1211.2837.

[72] S. Patra, "Neutrinoless double beta decay process in left-right symmetric models without scalar bidoublet," Phys.Rev. D87 (2013) no. 1, 015002, arXiv:1212.0612.

[73] R. L. Awasthi, M. Parida, and S. Patra, "Neutrino masses, dominant neutrinoless double beta decay, and observable lepton flavor violation in left-right models and $S O(10)$ grand unification with low mass $W_{R}, Z_{R}$ bosons," JHEP 1308 (2013) 122, arXiv:1302.0672.

[74] J. Barry and W. Rodejohann, "Lepton number and flavour violation in TeV-scale left-right symmetric theories with large left-right mixing," JHEP 1309 (2013) 153, arXiv:1303.6324.

[75] P. Bhupal Dev, S. Goswami, M. Mitra, and W. Rodejohann, "Constraining Neutrino Mass from Neutrinoless Double Beta Decay," Phys.Rev. D88 (2013) 091301, arXiv:1305.0056.

[76] W.-C. Huang and J. Lopez-Pavon, "On neutrinoless double beta decay in the minimal left-right symmetric model," Eur. Phys. J. C74 (2014) 2853, arXiv:1310.0265.

[77] P. S. Bhupal Dev, S. Goswami, and M. Mitra, "TeV Scale Left-Right Symmetry and Large Mixing Effects in Neutrinoless Double Beta Decay," Phys. Rev. D91 (2015) no. 11, 113004, arXiv: 1405.1399.

[78] S.-F. Ge, M. Lindner, and S. Patra, "New physics effects on neutrinoless double beta decay from right-handed current," JHEP 10 (2015) 077, arXiv:1508.07286.

[79] D. Borah and A. Dasgupta, "Neutrinoless Double Beta Decay in Type I+II Seesaw Models," JHEP 11 (2015) 208, arXiv:1509.01800.

[80] R. L. Awasthi, P. S. B. Dev, and M. Mitra, "Implications of the Diboson Excess for Neutrinoless Double Beta Decay and Lepton Flavor Violation in TeV Scale Left Right Symmetric Model," Phys. Rev. D93 (2016) no. 1, 011701, arXiv:1509.05387.

[81] M. Horoi and A. Neacsu, "Analysis of mechanisms that could contribute to neutrinoless double-beta decay," Phys. Rev. D93 (2016) no. 11, 113014, arXiv:1511.00670.

[82] G. Bambhaniya, P. S. B. Dev, S. Goswami, and M. Mitra, "The Scalar Triplet Contribution to Lepton Flavour Violation and Neutrinoless Double Beta Decay in Left-Right Symmetric Model," JHEP 04 (2016) 046, arXiv:1512.00440.

[83] P.-H. Gu, "Neutrinoless double beta decay in the left-right symmetric models for linear seesaw," JHEP 09 (2016) 152, arXiv:1512.01119.

[84] D. Borah and A. Dasgupta, "Charged lepton flavour violcxmation and neutrinoless double 
beta decay in left-right symmetric models with type I+II seesaw," JHEP 07 (2016) 022, arXiv: 1606.00378.

[85] R. L. Awasthi, A. Dasgupta, and M. Mitra, "Limiting the Effective Mass and New Physics Parameters from 0 $\nu \beta \beta$," arXiv: 1607.03835.

[86] D. Borah and S. Patra, "Universal Seesaw and $0 \nu \beta \beta$ in new 3331 left-right symmetric model," arXiv:1701.08675.

[87] F. F. Deppisch, C. Hati, S. Patra, P. Pritimita, and U. Sarkar, "Neutrinoless Double Beta Decay in Left-Right Symmetry with Universal Seesaw," arXiv:1701.02107.

[88] M. K. Parida and S. Patra, "Left-right models with light neutrino mass prediction and dominant neutrinoless double beta decay rate," Phys. Lett. B718 (2013) 1407-1412, arXiv: 1211.5000.

[89] S. Patra, A. Sarkar, and U. Sarkar, "Spontaneous Left-Right Symmetry Breaking in Supersymmetric Models with only Higgs Doublets," Phys. Rev. D82 (2010) 015010, arXiv:1003.5095.

[90] D. Borah and S. Patra, "Type III Seesaw and Dark Matter in a Supersymmetric Left-Right Model," arXiv:0910.0146.

[91] S. Patra, A. Sarkar, U. Sarkar, and U. Yajnik, "Spontaneous Parity Violation in a Supersymmetric Left-Right Symmetric Model," Phys. Lett. B679 (2009) 386-389, arXiv:0905.3220.

[92] H. Borgohain and M. K. Das, "Neutrinoless double beta decay and lepton flavour violation in broken $\mu-\tau$ symmetric neutrino mass models," arXiv:1705.00922.

[93] F. Ahmed, A. Neacsu, and M. Horoi, "Interference between light and heavy neutrinos for $0 \nu \beta \beta$ decay in the leftâĂŞright symmetric model," Phys. Lett. B769 (2017) 299-304, arXiv: 1701.03177.

[94] Riazuddin, R. E. Marshak, and R. N. Mohapatra, "Majorana Neutrinos and Low-energy Tests of Electroweak Models," Phys. Rev. D24 (1981) 1310-1317.

[95] P. B. Pal, "Constraints on a Muon - Neutrino Mass Around 100-kev," Nucl. Phys. B227 (1983) 237-251.

[96] R. N. Mohapatra, "Rare decays of the tau lepton as a probe of the left-right symmetric theories of weak interactions," Phys. Rev. D46 (1992) 2990-2995.

[97] V. Cirigliano, A. Kurylov, M. J. Ramsey-Musolf, and P. Vogel, "Lepton flavor violation without supersymmetry," Phys. Rev. D70 (2004) 075007, arXiv:hep-ph/0404233.

[98] V. Cirigliano, A. Kurylov, M. J. Ramsey-Musolf, and P. Vogel, "Neutrinoless double beta decay and lepton flavor violation," Phys. Rev. Lett. 93 (2004) 231802, arXiv:hep-ph/0406199.

[99] B. Bajc, M. Nemevsek, and G. Senjanovic, "Probing leptonic CP phases in LFV processes," Phys. Lett. B684 (2010) 231-235, arXiv:0911.1323.

[100] C.-H. Lee, P. S. Bhupal Dev, and R. N. Mohapatra, "Natural TeV-scale left-right seesaw mechanism for neutrinos and experimental tests," Phys. Rev. D88 (2013) no. 9, 093010 , arXiv: 1309.0774.

[101] D. Borah, S. Patra, and P. Pritimita, "Sub-dominant type-II seesaw as an origin of non-zero 
$\theta_{13}$ in SO(10) model with TeV scale $Z^{\prime}$ gauge boson," Nucl. Phys. B881 (2014) 444-466, arXiv: 1312.5885 .

[102] J. Chakrabortty, P. Ghosh, S. Mondal, and T. Srivastava, "Reconciling (g-2) and charged lepton flavor violating processes through a doubly charged scalar," Phys. Rev. D93 (2016) no. 11, 115004, arXiv:1512.03581.

[103] M. Lindner, M. Platscher, and F. S. Queiroz, "A Call for New Physics : The Muon Anomalous Magnetic Moment and Lepton Flavor Violation," arXiv:1610.06587.

[104] C. Bonilla, M. E. Krauss, T. Opferkuch, and W. Porod, "Perspectives for Detecting Lepton Flavour Violation in Left-Right Symmetric Models," arXiv:1611.07025.

[105] G. Ecker, W. Grimus, and H. Neufeld, "The Neutron Electric Dipole Moment in Left-right Symmetric Gauge Models," Nucl. Phys. B229 (1983) 421-444.

[106] J. F. Nieves, D. Chang, and P. B. Pal, "Electric Dipole Moment of the Electron in Left-right Symmetric Theories," Phys. Rev. D33 (1986) 3324-3328.

[107] J. M. Frere, J. Galand, A. Le Yaouanc, L. Oliver, O. Pene, and J. C. Raynal, "The Neutron electric dipole moment in left-right symmetric models," Phys. Rev. D45 (1992) 259-277.

[108] A. Maiezza and M. NemevÅąek, "Strong P invariance, neutron electric dipole moment, and minimal left-right parity at LHC," Phys. Rev. D90 (2014) no. 9, 095002, arXiv:1407.3678.

[109] J. Heeck and S. Patra, "Minimal Left-Right Symmetric Dark Matter," Phys. Rev. Lett. 115 (2015) no. 12, 121804, arXiv:1507.01584.

[110] C. Garcia-Cely and J. Heeck, "Phenomenology of left-right symmetric dark matter," arXiv:1512.03332. [JCAP1603,021(2016)].

[111] S. Patra, "Dark matter, lepton and baryon number, and left-right symmetric theories," Phys. Rev. D93 (2016) no. 9, 093001, arXiv:1512.04739.

[112] D. Borah, S. Patra, and S. Sahoo, "Subdominant leftâĂŞright scalar dark matter as origin of the 750 GeV di-photon excess at LHC," Int. J. Mod. Phys. A31 (2016) no. 17, 1650097, arXiv: 1601.01828.

[113] D. Borah, A. Dasgupta, and S. Patra, "Common Origin of 3.55 keV X-ray line and Gauge Coupling Unification with Left-Right Dark Matter," arXiv:1604.01929.

[114] D. Borah, A. Dasgupta, U. K. Dey, S. Patra, and G. Tomar, "Multi-component Fermionic Dark Matter and IceCube PeV scale Neutrinos in Left-Right Model with Gauge Unification," arXiv: 1704.04138.

[115] S. Patra and S. Rao, "Singlet fermion Dark Matter within Left-Right Model," Phys. Lett. B759 (2016) 454-458, arXiv:1512.04053.

[116] F. F. Deppisch, C. Hati, S. Patra, P. Pritimita, and U. Sarkar, "Implications of the diphoton excess on left-right models and gauge unification," Phys. Lett. B757 (2016) 223-230, arXiv: 1601.00952.

[117] T. Bandyopadhyay and A. Raychaudhuri, "Left-right model with TeV fermionic dark matter and unification," arXiv:1703.08125.

[118] C. ArbelĀąez, M. Hirsch, and D. Restrepo, "Fermionic triplet dark matter in an SO(10)-inspired left right model," arXiv:1703.08148.

[119] G. E. Volovik, "Dark matter from SU(4) model," Pisma Zh. Eksp. Teor. Fiz. 78 (2003) 1203-1206, arXiv:hep-ph/0310006. [JETP Lett.78,691(2003)]. 
[120] P.-H. Gu, "A Left-Right Symmetric Model for Neutrino Masses, Baryon Asymmetry and Dark Matter," Phys. Rev. D81 (2010) 095002, arXiv:1001.1341.

[121] K. S. Babu and R. N. Mohapatra, "Predictive neutrino spectrum in minimal SO(10) grand unification," Phys. Rev. Lett. 70 (1993) 2845-2848, arXiv:hep-ph/9209215.

[122] H. Georgi and S. L. Glashow, "Unity of All Elementary Particle Forces," Phys. Rev. Lett. 32 (1974) 438-441.

[123] T. G. Rizzo and G. Senjanovic, "Grand Unification and Parity Restoration at Low-Energies. 1. Phenomenology," Phys. Rev. D24 (1981) 704. [Erratum: Phys. Rev.D25,1447(1982)].

[124] D. Chang, R. N. Mohapatra, and M. K. Parida, "Decoupling Parity and SU(2)-R Breaking Scales: A New Approach to Left-Right Symmetric Models," Phys. Rev. Lett. 52 (1984) 1072.

[125] D. Chang, R. N. Mohapatra, and M. K. Parida, "A New Approach to Left-Right Symmetry Breaking in Unified Gauge Theories," Phys. Rev. D30 (1984) 1052.

[126] D. Chang, R. N. Mohapatra, J. Gipson, R. E. Marshak, and M. K. Parida, "Experimental Tests of New SO(10) Grand Unification," Phys. Rev. D31 (1985) 1718.

[127] B. Bajc and G. Senjanovic, "Radiative seesaw and degenerate neutrinos," Phys. Rev. Lett. 95 (2005) 261804, arXiv:hep-ph/0507169.

[128] S.-F. Ge and W. Rodejohann, "JUNO and Neutrinoless Double Beta Decay," arXiv: 1507.05514.

[129] F. F. Deppisch, T. E. Gonzalo, S. Patra, N. Sahu, and U. Sarkar, "Signal of Right-Handed Charged Gauge Bosons at the LHC?," Phys. Rev. D90 (2014) no. 5, 053014, arXiv: 1407.5384.

[130] S. Patra and P. Pritimita, "Post-sphaleron baryogenesis and $n-\bar{n}$ oscillation in non-SUSY SO(10) GUT with gauge coupling unification and proton decay," Eur. Phys. J. C74 (2014) no. 10, 3078, arXiv:1405.6836.

[131] P. S. Bhupal Dev, C.-H. Lee, and R. N. Mohapatra, "Leptogenesis Constraints on the Mass of Right-handed Gauge Bosons," Phys. Rev. D90 (2014) no. 9, 095012, arXiv:1408.2820.

[132] S. Bertolini, A. Maiezza, and F. Nesti, "Present and Future K and B Meson Mixing Constraints on TeV Scale Left-Right Symmetry," Phys.Rev. D89 (2014) 095028, $\operatorname{arXiv:1403.7112.~}$

[133] G. Beall, M. Bander, and A. Soni, "Constraint on the Mass Scale of a Left-Right Symmetric Electroweak Theory from the $K_{L}-K_{S}$ Mass Difference," Phys.Rev.Lett. 48 (1982) 848.

[134] S. Bertolini, T. Schwetz, and M. Malinsky, "Fermion masses and mixings in SO(10) models and the neutrino challenge to SUSY GUTs," Phys. Rev. D73 (2006) 115012, arXiv: hep-ph/0605006.

[135] F. Buccella, M. Chianese, G. Mangano, G. Miele, S. Morisi, and P. Santorelli, "A neutrino mass-mixing sum rule from SO(10) and neutrinoless double beta decay," JHEP 04 (2017) 004, arXiv:1701.00491.

[136] H. Fritzsch and P. Minkowski, "Unified Interactions of Leptons and Hadrons," Annals Phys. 93 (1975) 193-266.

[137] M. K. Parida, R. L. Awasthi, and P. K. Sahu, "Proton decay and new contribution to $0 \nu 2 \beta$ decay in $S O(10)$ with low-mass $Z$ ' boson, observable $n-\bar{n}$ oscillation, lepton flavor violation, and rare kaon decay," JHEP 01 (2015) 045, arXiv:1401.1412. 
[138] P. S. B. Dev and R. N. Mohapatra, "TeV Scale Inverse Seesaw in SO(10) and Leptonic Non-Unitarity Effects," Phys. Rev. D81 (2010) 013001, arXiv:0910.3924.

[139] R. Lal Awasthi and M. K. Parida, "Inverse Seesaw Mechanism in Nonsupersymmetric SO(10), Proton Lifetime, Nonunitarity Effects, and a Low-mass Z' Boson," Phys. Rev. D86 (2012) 093004, arXiv:1112.1826.

[140] Particle Data Group, K. A. Olive et al., "Review of Particle Physics," Chin. Phys. C38 (2014) 090001.

[141] P. Pritimita, N. Dash, and S. Patra, "Neutrinoless Double Beta Decay in LRSM with Natural Type-II seesaw Dominance," JHEP 10 (2016) 147, arXiv:1607.07655.

[142] M. C. Gonzalez-Garcia, M. Maltoni, J. Salvado, and T. Schwetz, "Global fit to three neutrino mixing: critical look at present precision," JHEP 12 (2012) 123, arXiv:1209.3023.

[143] G. L. Fogli, E. Lisi, A. Marrone, D. Montanino, A. Palazzo, and A. M. Rotunno, "Global analysis of neutrino masses, mixings and phases: entering the era of leptonic CP violation searches," Phys. Rev. D86 (2012) 013012, arXiv:1205.5254.

[144] M. C. Gonzalez-Garcia, M. Maltoni, and T. Schwetz, "Updated fit to three neutrino mixing: status of leptonic CP violation," JHEP 11 (2014) 052, arXiv:1409.5439. 